\title{
On Carrier Sensing Accuracy and Range Scaling Laws in Nakagami Fading Channels
}

\author{
Yue Wang, ${ }^{1}$ Liang Chen, ${ }^{2}$ Li Zhang, ${ }^{3}$ Haifeng Li, ${ }^{1}$ Huaihu Cao, ${ }^{1}$ and Jianming Zhu ${ }^{1}$ \\ ${ }^{1}$ School of Information, Central University of Finance and Economics, Beijing, China \\ ${ }^{2}$ College of Information Engineering, Shenzhen University, Shenzhen, China \\ ${ }^{3}$ School of Software, Beijing University of Technology, Beijing, China \\ Correspondence should be addressed to Liang Chen; lchen@szu.edu.cn
}

Received 6 April 2017; Revised 8 August 2017; Accepted 24 August 2017; Published 26 November 2017

Academic Editor: Xianfu Lei

Copyright (C) 2017 Yue Wang et al. This is an open access article distributed under the Creative Commons Attribution License, which permits unrestricted use, distribution, and reproduction in any medium, provided the original work is properly cited.

\begin{abstract}
We make a detailed study on carrier sensing of 802.11 in Nakagami fading channels. We prove that to maximize sensing accuracy, the optimal channel accessing probability is solely determined by the path-loss SIR (Signal to Interference Ratio). We define $p_{\text {fail }}{ }^{-}$ interference range and $p_{\text {busy }}$-carrier sensing range for fading channels and prove that their scaling laws in Nakagami fading channels are similar to those in the static channel. The newly derived theoretical results show a unified property between the static and fading channels. By extensive simulations, we reveal that fading depresses the probability of a dominating transmission state, and therefore it can mitigate severe hidden and exposed terminal problems, but fading harms the average sensing accuracy for an optimally adjusted carrier sensing threshold.
\end{abstract}

\section{Introduction}

IEEE 802.11 has gained broad and far-reaching applications in our life, due to its agile deployment and cheap maintenance. A lot of previous works in wireless network models assume that signal strength is invariant over time in their study. It may be enough to consider the simple static channel in indoor $\mathrm{WiFi}$. However, multipath fading comes into the picture when we deal with the outdoor environment where signal strength varies with time because stations are moving or surroundings are changing.

Although some network models have begun to incorporate multipath fading, and measurements and high-fidelity simulations show it is necessary to do so, some basic parts are missing, which are of concern to networking people, such as understanding the interplay between interference and carrier sensing, and the extension of interference/carrier sensing ranges to fading channels and their properties.

In this work, we study a two-link topology so as to examine basic principles as done in $[1,2]$. Compared with related works (Section 2), we present some new insights on how and why the randomness of fading may affect carrier sensing, rather than incorporating fading into models and simply making calculations. We deal with the Nakagami fading model, which represents a general family of fading channels, and carry out simulations for the main bit rates of $802.11 \mathrm{a} / \mathrm{b} / \mathrm{g} / \mathrm{n}$. The broad settings of parameters make our results confident. Note that our work differs from existing communication papers on fading, and the paper mainly talks about the interplay between interference and carrier sensing, which is less addressed in the literature.

Specifically, we make the following contributions.

1.1. Interplay between Carrier Sensing and Interference: Optimal, Case Study, and Average Carrier Sensing Accuracies (Section 4). We prove that, in order to maximize carrier sensing accuracy in Nakagami fading channels, whether the transmitter accesses the channel or not is solely determined by whether the path-loss SIR (Signal to Interference Ratio) is larger than or less than the SNR threshold, independent of channel time-variations (Theorem 3).

Simulations show that the four transmission states of (idle, success), (idle, failure), (busy, success), and (busy, failure) are mixed together in fading channels. Since fading caused randomness depresses the probability of a dominating state, it can mitigate severe hidden and exposed terminal problems. 
We further study the average sensing accuracy averaging on different interferer locations within a disk plane. Intensive simulations show that fading degrades the average sensing accuracy for an optimally adjusted carrier sensing threshold.

\subsection{Definitions of Interference/Carrier Sensing Range and} Their Scaling Laws in Nakagami Fading Channels (Section 5). We define $p_{\text {fail }}$-interference range and $p_{\text {busy }}$-carrier sensing range, as interference and carrier sensing are mentioned in a probability manner in fading channels. We prove the range scaling laws of fading channels, which are similar to the ones of the static channel (Theorems 6 and 8). Besides the application to visualize channel conditions, the scaling laws make it enough to experiment on one topology only rather than trying all different topologically relevant parameters one by one.

The paper is organized as follows. Section 2 discusses the related work and highlights our difference. Section 3 presents the system model, symbols, conventions, and assumptions. Section 4 studies the fading effects on carrier sensing considering the interplay with interference. Section 5 defines interference range and carrier sensing range for fading channels and proves their scaling laws and applications. Finally, Section 6 concludes the paper.

\section{Related Works}

Multipath fading needs to be considered in order to model 802.11 accurately. We make a comprehensive review of related works. Here we first summarize some representative ones of them and then highlight the difference of ours.

2.1. Literature Review. Since the last century, there have been some early works on the capture effect of multipath fading. Hansen and Meno derived the distribution function of reception power considering both lognormal shadowing and Rayleigh fading [3]. Sowerby and Williamson calculated the outage probability (i.e., packet reception failure probability) of a wanted communication in face of multiple interferers in the Rayleigh fading channel based on the capture threshold model or the SNRT (Signal to Noise Ratio Threshold) model with the independence assumption [4]. Yao and Sheikh extended the result in [4] to the Rician fading [5]. These works did not consider carrier sensing since they talk about a cellular cell.

In this century, multipath fading is incorporated into analysis models of CSMA, evolving from simplified models to complex models, from one WLAN to an ad hoc network scenario, from Rayleigh fading to a general fading model.

The first class of 802.11 modelling works assumed perfect carrier sensing and only studied fading effect on capture. Perfect carrier sensing means that all ongoing transmissions on the channel can be sensed by a ready-to-transmit wireless node and carrier sensing accuracy is not influenced by fading. Kim and Lee analyzed the CSMA/CA throughput under multistation interference in composite Rayleigh and shadowing channels [6], where they used the SNRT reception model and the carrier sensing mechanism of exchanging of RTS and CTS. Song et al. incorporated fading errors and extended Bianchi's CSMA model for saturated traffic [7]. Daneshgaran et al. further analyzed throughput performance for unsaturated traffic in the Rayleigh fading channel [8]. Leonardo and Yacoub also analyzed throughput performance of unsaturated traffic, but they provided an extensive calculation for the Hoyt, Rice, and Nakagami- $m$ fading channels [9]. In contrast to the above works dealing with a symmetric WLAN cell, Sheng and Vastola proposed a model for an ad hoc network in fading channels [10], by extending Chang et al.'s model in the static channel [11].

The second class of 802.11 modelling works considered imperfect carrier sensing, but they assumed a naive capture mechanism: two transmissions will collide if and only if they overlap in time. Early researchers studied the performance of nonpersistent [12], 1-persistent [13], and p-persistent CSMA [14] under imperfect carrier sensing. Chong et al. analyzed the throughput and delay performance of CSMA/CA as a function of sensing error probability [15]. Sheng and Vastola incorporated the outage probability of carrier sensing into Bianchi's model for the Rayleigh channel [16], where sensing error was determined by fading, instead of a predefined constant [12-14] or due to improperly set carrier sensing thresholds [15] assumed in previous works. The above works dealt with a symmetric WLAN cell and verified their analysis by simulation. Recently, Kai and Liew noted the partial carrier sensing relationship in their real experiment [17], and they said, "the carrier sensing relationship between the links are often probabilistic and can vary dynamically over time. This is the case even if the distance between the links is fixed and there is no drastic change in the environment," suggesting that imperfect carrier sensing needs to be considered carefully.

The third class of 802.11 modelling works studied the combined effects of fading on capture and carrier sensing. Based on Bianchi's model, Sheng and Vastola [18] modelled the throughput of 802.11 DCF in a symmetric circular topology and jointly considered the effects of Rayleigh fading on capture and carrier sensing. They noted that a fixed carrier sense threshold might not achieve the best performance at different network radii. Dai and Yamao proposed a probability analysis method that can predict packet delivery ratio for a multihop linear topology under fading environment, and they analyzed the impact of hidden terminal caused intraflow interference and compared two carrier sensing thresholds $(-81 \mathrm{dBm}$ and $-85 \mathrm{dBm}$ ) [19]. Mittag and Hartenstein simulated V2V networks and they concluded that fading had only a slight impact on the effectiveness of CSMA [20]. Schumacher and Tchouankem showed that the severity of fading substantially influenced packet delivery based on their collected empirical data of highway traffic [21]. However, they used a fixed carrier sensing threshold and did not study its impact.

Recently, stochastic geometry [22] is noted as a powerful tool for analyzing and planning of a large dense CSMA network. We name some of them in the following. In [23], Baccelli et al. used the Matern hard-core process [22] to model the CSMA protocol to study maximum network throughput. The Matern hard-core process is a thinning of the Poisson point process and it guarantees that each transmitter in the center of a disc contains no other transmitters than 


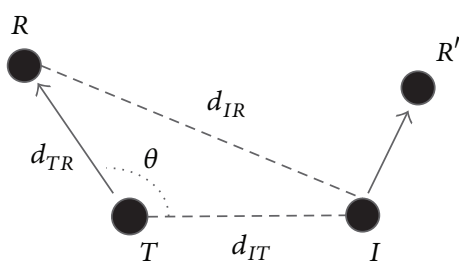

(a)

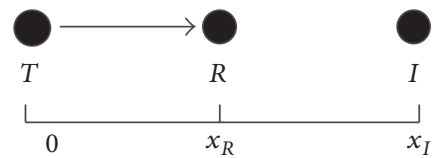

(b)

Figure 1: (a) General topology of two links. (b) Line topology.

itself, which is suitable to model carrier sensing. A fixed carrier sensing range that avoids collisions was used in the paper. In [24], also based on the Matern process, Nguyen et al. derived the closed-form formulas for the probability of coverage of the network and for the average throughput per user for a dense IEEE 802.11 network. A constant carrier sensing range of $300 \mathrm{~m}$ was used in the simulation. In [25], Haenggi proposed a unified framework that incorporates the fading process into the point process model (the point Poisson process was used) and he derived the formulas for node connectivity, broadcast reliability, broadcast transport capacity, and so forth in Nakagami- $m$ fading channels. No carrier sensing was considered in the paper since it studied the broadcast issues from a node. In [26], Haenggi and Ganti derived the interference statistics considering a constant carrier sensing range for the network topologies from lattices to homogeneous and clustered Poisson models to general motion-invariant ones. In [27], Kaynia et al. considered the performance of the ALOHA and CSMA MAC protocols in wireless ad hoc networks in the presence of fading. In their network model, packets belonging to specific transmitters arrive randomly in space and time according to a 3D Poisson point process. They found that the introduction of fading is added to the hidden and exposed node problems of CSMA, resulting in an up to $75 \%$ increase in the outage probability. In [28], Yang et al. devised a carrier sensing range in the presence of fading such that two nodes cannot sense each other larger than a certain probability. And they derived the upper bound of outage probability which has a functional relation to the carrier sensing range (see Theorem 1, [28]). In [29], Elsawy and Hossain proposed a modified hard-core point process to mitigate the node intensity underestimation flaw for any fading environment. As shown by their analysis, decreasing carrier sensing threshold decreases the intensity of simultaneously active transmitters as well as the hidden node problem, and there exists an optimal carrier sensing threshold which depends on the operating conditions of the network. In [30], Alfano et al. extended previous stochastic geometry models of CSMA networks and obtained throughput distributions, in addition to spatial averages. They observed that the carrier sensing threshold has a dramatic impact on the spatial fairness among the nodes. A large sensing threshold, while increasing transmission probability, can also cause strong interference and make a significant fraction of APs (those in unfavorable topological conditions) experience throughput starvation. Besides, they found that higher diversity in the fading distribution increases the spatial fairness and alleviates the starvation.
2.2. The Difference of Our Work. Although there are some works addressing carrier sensing in CSMA under fading environment, our work made one more step to discuss in detail the carrier sensing issue in fading channels.

First, many previous works focused on incorporating fading into CSMA models and getting accurate model prediction, but the interplay mechanism between interference and carrier sensing in fading channels was not thoroughly discussed, and the results might not be consistent sometimes, depending on the used network topologies or channel conditions. We want to focus on and give a clear understanding on carrier sensing solely.

Second, we study this issue from the micro-view perspective and focused on carrier sensing accuracy instead of the whole CSMA. Although the two-link topology in our work is much simpler than that in the stochastic geometry works, we revealed some nontrivial results. For instance, we found by case study that fading can depress the probability of a dominating transmission state, and in some scenarios it can mitigate severe hidden or exposed terminal problems when they are significant (see Figure 3). The case of the average sensing accuracy also shows that fading tends to moderate the sensing accuracy. For a carrier sensing threshold set inadequately, fading can upgrade or degrade the sensing accuracy (see Figure 5), while for the optimally adjusted carrier sensing threshold, we found by extensive simulations (see Appendix A.2) that the average sensing accuracy in the static channel is always higher than that in fading channels, and their difference is more pronounced when the channel variation becomes larger.

Third, we worked out some new theoretical results. We proved the condition of optimal channel accessing in fading channels. And we extended the definition of interference/carrier sensing ranges to fading channels and proved their scaling laws.

\section{System Model}

3.1. Experimental Topologies. We consider a two-link topology in Figure 1. The target link is $T-R$ (a) where $T$ is the transmitter and $R$ is the receiver, and the interfering link is $I$ $R^{\prime}$ where $I$ is the interfering transmitter, called the interferer. $d_{T R}, d_{I R}$, and $d_{I T}$ are defined as the link distance, interference distance, and carrier sensing distance, respectively. $d_{I R}$ is determined by $d_{T R}, d_{I T}$, and their angle $\theta$. Figure 1(b) is a simplified scenario of (a) where $T, R$, and $I$ are on a line, and their coordinates are $0, x_{R}$, and $x_{I}$, respectively. Here, we fix $x_{R}$ to be a positive value and $x_{I}$ can be positive or negative. 
The two simple topologies are usually used as the first step of discussion (e.g., in $[1,2])$ as we did here.

3.2. Channel Models. Radio propagation consists of three components: path loss, shadowing, and multipath fading (it applies to signal and interference; for simplicity, we illustrate the following concepts only by signal). Path loss is a deterministic large-scale decay of reception power, which can be expressed by

$$
\bar{s} \propto \frac{P_{t}}{d^{\alpha}} .
$$

Here $\bar{s}$ denotes the average signal reception power from a transmitter with the transmission power $P_{t}$ to a receiver of distance $d$ away. $\alpha$ is called path-loss exponent, typically 2 to 5 depending on the environment ( 2 in the Friis or free space model and 4 in the two-ray ground model). The typical $\alpha$ in our simulations is set to 3 [2].

Shadowing is a lognormal statistical variation from place to place due to obstacles and reflections, and the standard deviation $\sigma$ is typically $4-12 \mathrm{~dB}$.

Multipath fading (in short, fading) causes time-varying fine-grained statistical variations because of the mobility of transmitters, receivers, interferers, or the surrounding environment. There are many fading models of signal strength (i.e., square root of power), such as Rayleigh, Rice, Hoyt, Nakagami, and Weibull models. Experiments showed that signal strength can be described by the family of Nakagami-m distribution, where $m$ is an environment dependent parameter [31]. Fading is less severe when $m$ gets larger and there is no fading at all when $m$ goes to infinity. As special cases, Nakagami distribution turns into unilateral Gaussian distribution when $m=0.5$ and Rayleigh distribution when $m=1$. Besides, Hoyt, Rice, and Weibull distributions can be approximated by Nakagami distribution as well [9, 32].

In the Nakagami fading model, the reception power $S$ follows the Gamma distribution, with the probability density function (pdf) being

$$
f_{S}(s)=\frac{m^{m}}{\Gamma(m) \bar{s}^{m}} \cdot s^{m-1} e^{-m s / \bar{s}},
$$

where $\bar{s}$ is the average signal reception power calculated by path loss and shadowing. The signal power follows exponential distribution (i.e., Rayleigh fading) when $m=1$, with the pdf being

$$
f_{S}^{\text {Ray }}(s)=\frac{1}{\bar{s}} \cdot e^{-s / \bar{s}}
$$

Likewise, we define the pdfs of interference power at the receiver, $f_{I}(i)$ and $f_{I}^{\text {Ray }}(i)$, respectively.

3.3. Reception Models. There are two conditions for a packet to be decoded correctly. First, the reception power of the packet $s$ must be larger than or equal to the reception sensitivity $s_{0}$; that is, $\bar{s} \geq s_{0}$. Second, the signal to noise ratio (SNR) should be sufficiently large for the packet to be decoded correctly. And there are two reception models, that is, the BER (Bit Error Rate) model and the SNRT (Signal to Noise Ratio Threshold) model.
3.3.1. BER Model. In this model, the bit error rate $p_{e, v}$ under a modulation scheme with bit rate $v$ is a certain function of the SNR $\beta$. For example, the bit error rate of $802.11 \mathrm{~b} 1 \mathrm{Mbps}$ is $p_{e, 1}(\beta)=0.5 e^{22 \beta}$.

The packet success ratio is determined by the bit error rate and the number of bits $n$ of the packet, which is

$$
p_{\text {suc }, v}(\beta)=\left(1-p_{e, v}(\beta)\right)^{n} \text {. }
$$

In this work, we consider the main 802.11 standards and bit rates: $802.11 \mathrm{a} / \mathrm{g}$ 's $\{6,9,12,18,24,36,48,54\} \mathrm{Mbps}$; $802.11 b / g$ 's $\{1,2,5.5,11\}$ Mbps; 802.11n's $\{6.5,13,19.5,26,39$, $52,58,65\}$ Mbps. Here we use the notation $802.11 \mathrm{a} / \mathrm{g}(802.11$ $\mathrm{b} / \mathrm{g}$ ) to denote the common bit rates of 802.11a and $802.11 \mathrm{~g}$ (802.11b and $802.11 \mathrm{~g}$ ), respectively, because $802.11 \mathrm{~g}$ inherits from 802.11a and 802.11b. And we only consider the basic settings of 802.11n: MCS0-MCS7 in 802.11n@20 MHz.

3.3.2. SNRT Model. It is an approximation of the BER model. It says that a packet can be received as long as its $\operatorname{SNR} \beta$ is beyond the SNR threshold $\beta_{0, v}$; that is,

$$
\beta \geq \beta_{0, v} .
$$

The SNR threshold $\beta_{0, v}$ is usually determined when $p_{\text {suc }, v}\left(\beta_{0, v}\right)$ is equal to 0.5 (note that $\beta_{0, v}$ is a function of $n$, but it is insensitive to $n$; our simulation shows that the average SNR threshold increases by $1.88 \mathrm{~dB}$ when $n$ increases from 2048 to 63848 (MTU bit size of 802.11)).

3.3.3. Comparison of Two Reception Models. Figure 2 shows packet success ratios for the bit rates in $802.11 \mathrm{a} / \mathrm{g}, 802.11 \mathrm{~b} / \mathrm{g}$, and $802.11 n$ when $\alpha$ is 3 and $n=2048$ (256 bytes). We test the topology of Figure 1(b), fixing $x_{R}$ to $10 \mathrm{~m}$ and varying $x_{I}$ to generate different SNRs. One can see that there is a sudden shift of packet success ratio when $x_{I}$ (and the corresponding $\mathrm{SNR}$ ) is beyond a certain value.

3.4. Symbols and Conventions. Symbols section shows basic symbols and Table 1 shows simulation parameters. The receive sensitivity $s_{0, v}$ is from Cisco Aironet 1040 Series Wireless Access Point [33]. The SNR threshold $\beta_{0, v}$ is calculated for a 256-byte packet, used in the SNRT model. For simple notations, $\beta_{0, v}$ is abbreviated to $\beta_{0}$ and $p_{\text {suc, } v}$ to $p_{\text {suc }}$ in the context without confusion.

We discriminate SNR against SIR here. SNR (some papers use SINR) refers to the ratio of signal power to noise power and the noise is the sum of thermal noise and interference. SIR refers to the ratio of signal power to interference power only. Thus, we use the name SNR threshold instead of SIR threshold. When thermal noise is small and ignored, we can compare an SIR to the SNR threshold.

The unit of power is $\mathrm{mW}$, and the unit of SNR or SIR is the original ratio in all the following equations, but we may use $\mathrm{dBm}$ and $\mathrm{dB}$ to show results in figures.

3.5. Simulation Settings and Assumptions. In the simulation, we let transmitters and interferers transmit equal-length packets simultaneously and consecutively, and we also isolate contention resolution mechanisms (e.g., backoff) from the problem under consideration. This setting is just right for 


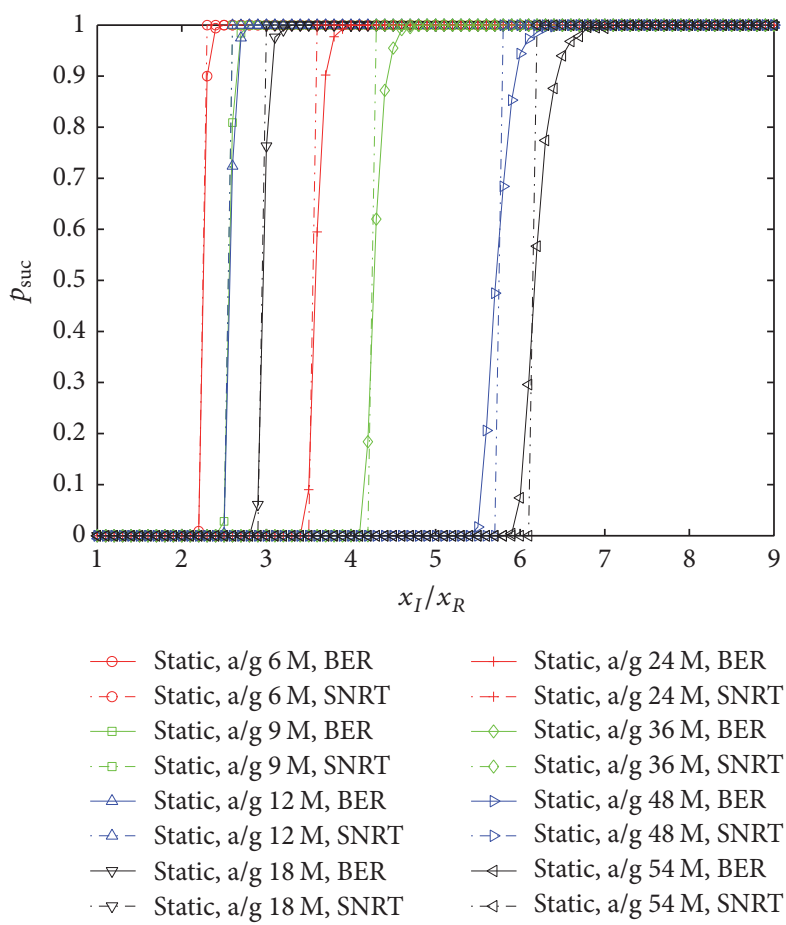

(a) $802.11 \mathrm{a} / \mathrm{g}$ (9 M and $12 \mathrm{M}$ are overlapped)

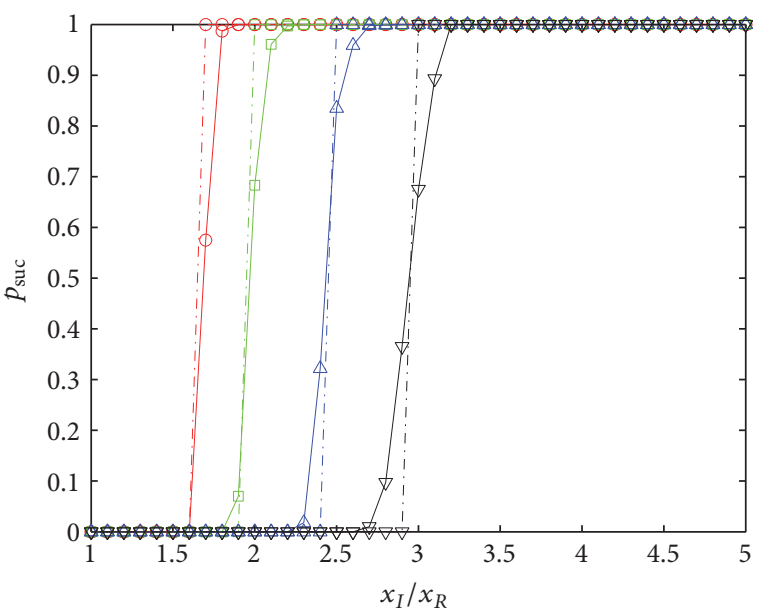

$$
\begin{aligned}
& - \text { Static, b/g } 1 \mathrm{M}, \mathrm{BER} \quad-\square-\text { Static, b/g } 2 \mathrm{M}, \mathrm{SNRT} \quad \nabla \quad \text { Static, b/g } 11 \mathrm{M}, \mathrm{BER} \\
& - \text { - Static, b/g } 1 \mathrm{M}, \text { SNRT } \triangle \text { Static, b/g } 5.5 \mathrm{M}, \mathrm{BER} \quad-\nabla-\text { Static, b/g } 11 \mathrm{M}, \mathrm{SNRT} \\
& \square \text { Static, b/g } 2 \mathrm{M}, \mathrm{BER} \quad-\triangle-\text { Static, b/g } 5.5 \mathrm{M} \text {, SNRT }
\end{aligned}
$$

(b) $802.11 \mathrm{~b} / \mathrm{g}$

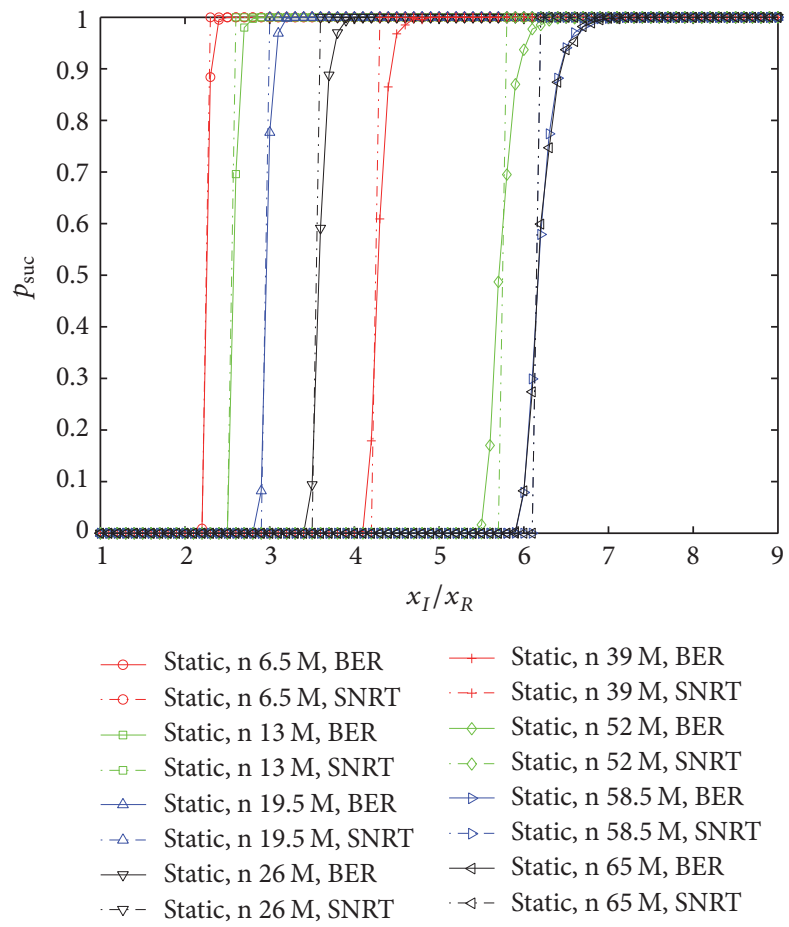

(c) $802.11 \mathrm{n}$

Figure 2: Packet success ratios of $802.11 \mathrm{a} / \mathrm{g}, 802.11 \mathrm{~b} / \mathrm{g}$, and $802.11 \mathrm{n}$ in the static channel ( $\alpha$ is 3; packet size is 256 bytes), comparing the BER and SNRT reception models.

our research problem: to understand the basic principles of carrier sensing in fading channels.

We ported ns-3 BER codes of all modulation schemes into our simulation, which was shown close to real measurement $[34,35]$, and implemented by ourselves the SNRT reception model for the purpose of comparison. To be rigorous, we usually use the BER reception model in simulations unless stated otherwise. We also ported ns-3 Nakagami fading codes into our simulation. The simulation codes are put on GitHub [36]. 

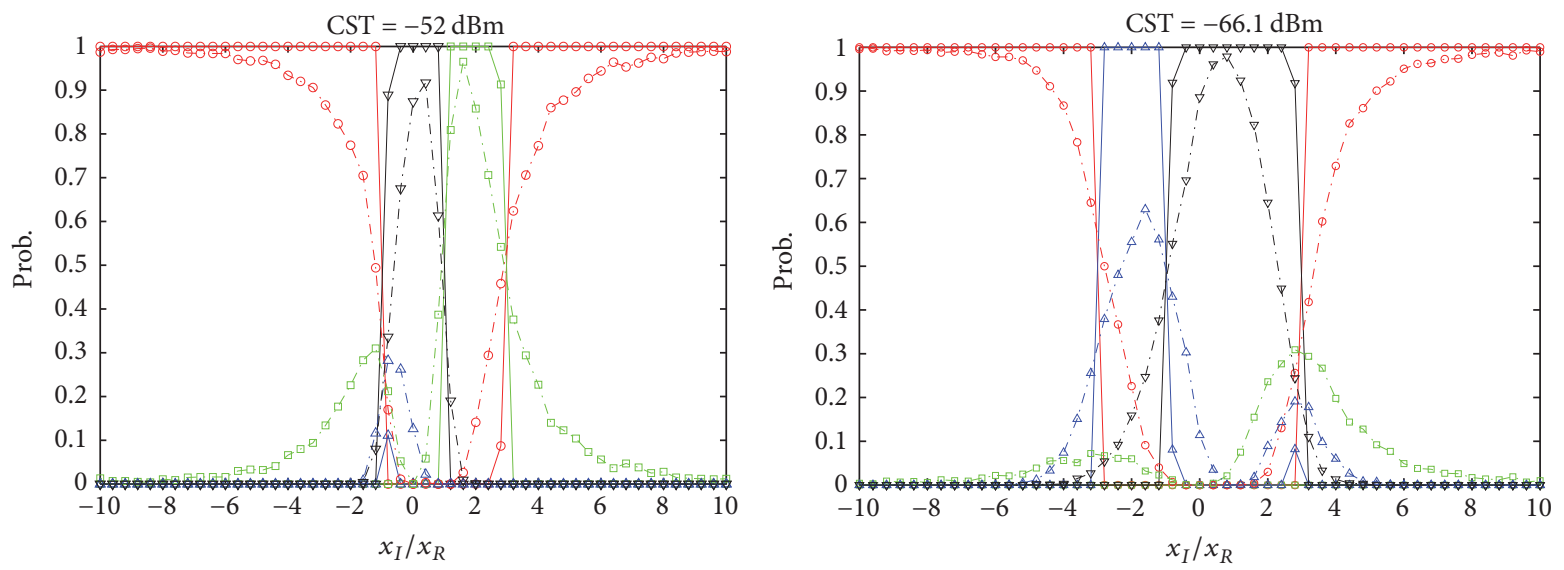

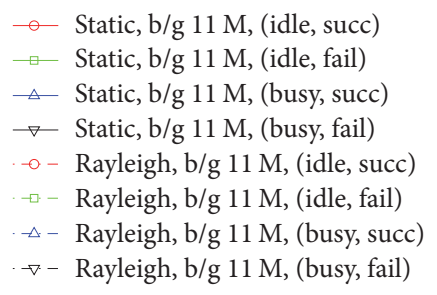

(a) Aggressive CST $=\bar{s}=-52 \mathrm{dBm}$

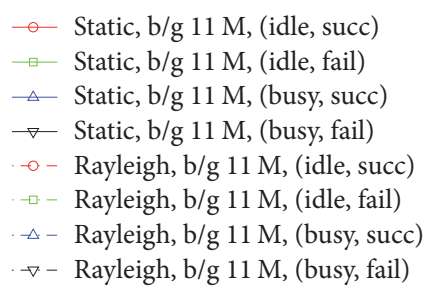

(b) Conservative $\operatorname{CST}=\bar{s} /\left(1+\beta_{0}^{1 / \alpha}\right)^{\alpha}=-66.1 \mathrm{dBm}$

Figure 3: Probabilities of transmission states in $802.11 \mathrm{~b} / \mathrm{g} 11 \mathrm{Mbps}$ as $x_{I} / x_{R}$ increases, comparing the static and Rayleigh channel $(\alpha=3$, $x_{R}=10 \mathrm{~m}$, and packet size is 256 bytes).

Transmission power is fixed to $15 \mathrm{dBm}$ and thermal noise is fixed to $-95.6 \mathrm{dBm}$. The packet size is set to 256 bytes unless stated otherwise.

We assume that signal power $S$, interference power $I$, and carrier sensed power $C$ are independent. It is a reasonable assumption as they go through different propagation paths. When the three variables are in strict proportion to each other (i.e., $S=k_{1} I=k_{2} C$ ), in other words, they are fully correlated, the analysis is reduced to the static channel case. And the other correlation degrees are somewhat in between. In spite of the above assumptions, we will see that some essential principles can be revealed.

To facilitate computation, we assume that reception power is invariant within the transmission time of a packet. It is reasonable for low speed scenarios (e.g., pedestrian speed), and the relative speed between adjacent vehicles may not be high in V2V networks.

\section{Carrier Sensing in Nakagami Fading Channels}

A transmitter accesses the channel when it is sensed to be idle. A common practice of 802.11 is to use a fixed carrier sensing threshold and the channel is deemed to be idle when the sensed interference power at the transmitter is below the threshold. In this section, we first derive the optimal channel access (idle) probability, and then we study the random behaviors of legacy carrier sensing, focusing on its accuracy.

4.1. Optimal Channel Access. Let $p_{\text {idle }}$ be the channel idle (or access) probability and let $p_{\text {suc }}$ be packet success ratio. Assume that carrier sensing power $C$ (at the transmitter), interference
TABLE 1: Simulation parameters.

\begin{tabular}{lcccc}
\hline \multicolumn{5}{c}{$802.11 \mathrm{a} / \mathrm{g}$} \\
\hline$v$ (Mbps) & 6 & 9 & 12 & 18 \\
\hline$s_{0}(\mathrm{~mW})$ & $3.98 E-9$ & $3.98 E-9$ & $3.98 E-9$ & $-3.98 E-9$ \\
$\beta_{0}$ (ratio) & 1.95 & 3.75 & 3.90 & 7.50 \\
\hline$v$ (Mbps) & 24 & 36 & 48 & 54 \\
\hline$s_{0}(\mathrm{~mW})$ & $5.01 E-9$ & $7.94 E-9$ & $2.51 E-8$ & $3.16 E-8$ \\
$\beta_{0}$ (ratio) & 17.1 & 34.9 & 104 & 137 \\
\hline \multicolumn{5}{c}{$802.11 \mathrm{~b} / \mathrm{g}$} \\
\hline$v$ (Mbps) & 1 & 2 & 5.5 \\
\hline$s_{0}(\mathrm{~mW})$ & $1.26 E-9$ & $1.26 E-9$ & $1.26 E-9$ & $2.51 E-9$ \\
$\beta_{0}$ (ratio) & 0.331 & 0.916 & 2.90 \\
\hline \multicolumn{5}{c}{$802.11 \mathrm{n}$} \\
\hline$v$ (Mbps) & 6.5 & 13 & 19.5 \\
\hline$s_{0}$ (mW) & $2.51 E-9$ & $3.16 E-9$ & $3.98 E-9$ & $6.31 E-9$ \\
$\beta_{0}$ (ratio) & 1.95 & 3.90 & 7.50 & 17.1 \\
\hline$v$ (Mbps) & 39 & 52 & 58.5 & 65 \\
\hline$s_{0}$ (mW) & $1.59 E-8$ & $3.98 E-8$ & $6.31 E-8$ & $7.94 E-8$ \\
$\beta_{0}$ (ratio) & 34.9 & 104 & 137 & 137 \\
\hline
\end{tabular}

power $I$, and signal reception power $S$ (both at the receiver) are all independent. The probabilities of the four transmission states are shown in Table 2.

We use the following optimization model to calculate the optimal $p_{\text {idle }}\left(p_{\text {idle }}^{*}\right)$ :

$$
\begin{aligned}
\max & w p_{\text {idle }} p_{\text {suc }}+(1-w)\left(1-p_{\text {idle }}\right)\left(1-p_{\text {suc }}\right) \\
\text { s.t. } & 0 \leq p_{\text {idle }} \leq 1,
\end{aligned}
$$


TABle 2: Probabilities of four transmission states under independence assumption.

\begin{tabular}{lcc}
\hline & Successful reception & Failed reception \\
\hline Idle channel & $p_{\text {idle }} p_{\text {suc }}$ & $p_{\text {idle }}\left(1-p_{\text {suc }}\right)$ \\
Busy channel & $\left(1-p_{\text {idle }}\right) p_{\text {suc }}$ & $\left(1-p_{\text {idle }}\right)\left(1-p_{\text {suc }}\right)$ \\
\hline
\end{tabular}

where $w$ is a weight between 0 and 1 to balance two parts of accuracy. We get

$$
p_{\text {idle }}^{*}= \begin{cases}0, & \text { if } p_{\text {suc }}<1-w \\ 1, & \text { if } p_{\text {suc }} \geq 1-w\end{cases}
$$

It suggests that a link should transmit when $p_{\text {suc }}$ is above $1-w$ and avoid transmitting, otherwise. When $w=1, p_{\text {idle }}^{*}=$ 1 , which maximizes throughput but sacrifices fairness. When $w$ is 0.5 , the objective function value is half of carrier sensing accuracy that is defined as

$$
p_{\text {idle }} p_{\text {suc }}+\left(1-p_{\text {idle }}\right)\left(1-p_{\text {suc }}\right) \text {. }
$$

The following result relates optimal channel access probability to the path-loss SIR.

Definition 1. The path-loss SIR $\bar{\beta}$ is defined as the average signal power to the average interference power, calculated by large-scale path-loss and shadowing models.

Lemma 2. In the Nakagami fading model, $p_{s u c}$ is always 0.5 when the path-loss SIR $\bar{\beta}$ is equal to the SNR threshold $\beta_{0}$, based on the SNRT model and ignoring receive sensitivity $s_{0}$ and thermal noise.

Proof. See Appendix A.1.

Theorem 3. (This argument is proved under the assumption of independent powers, using the SNRT model and ignoring receive sensitivity and thermal noise.) In Nakagami fading channels,

$$
p_{\text {idle }}^{*}= \begin{cases}0, & \text { if } \bar{\beta}<\beta_{0}, \\ 1, & \text { if } \bar{\beta} \geq \beta_{0} .\end{cases}
$$

Proof. When $w=0.5, p_{\text {suc }}=0.5$ at $\bar{\beta}=\beta_{0}$ (by Lemma 2), and the argument immediately follows.

Remark 4. This theorem shows that there exists a simple and unified way to access the channel optimally no matter what fading severity.

4.2. For Legacy Carrier Sensing in 802.11. However, it is difficult in practice for a transmitter to know path-loss SIR and access the channel optimally. Next, we consider the legacy carrier sensing of 802.11 where a fixed carrier sensing threshold (abbr. CST) is used.

4.2.1. Case Study. Since the carrier sensed interference power $C$ is a random variable,

$$
p_{\text {idle }}=\operatorname{Prob}\left\{C<c_{0}\right\},
$$

where $c_{0}$ is the CST.
We consider two commonly used CSTs [1]. Aggressive CST is set equal to the average reception power $\bar{s}$ where the carrier sensing range in the static channel is equal to link distance, liable to cause the hidden terminal problem, while conservative CST is set to $\bar{s} /\left(1+\beta_{0}^{1 / \alpha}\right)^{\alpha}$ where the carrier sensing range in the static channel is equal to link distance plus interference range, liable to cause the exposed terminal problem. The carrier sensing range and interference range here are defined in the static channel for rough estimation of distance, and we will redefine them in fading channels in the next section.

Due to space limitation, we mainly show the results of $802.11 \mathrm{~b} / \mathrm{g} 11 \mathrm{Mbps}$ in the Rayleigh channel, and similar behaviors were found for the other bit rates.

First, transmission states mix together in fading channels. Unlike in the static channel where the channel is either idle or busy and a transmission is either successful or failed, the random nature of fading makes multiple transmission states occur simultaneously (Figure 3 ).

Second, transition trends of transmission states in fading channels follow those in the static channel (Figure 3). Packet success ratio increases as the interferer moves away from the receiver, and channel idle probability increases as the interferer moves away from the transmitter-the essence still holds in fading channels.

Third, fading depresses the probability of a dominating transmission state. In the case of aggressive CST (Figure 3(a)), when $x_{I} / x_{R}$ increases from 1 to 3 , the hidden terminal problem in the fading channel becomes less severe than that in the static channel due to nonzero $p_{\text {suc }}$ (Figure $4(\mathrm{a})$ ). However, when $x_{I} / x_{R}$ goes from 4 to 8 , the hidden terminal problem vanishes in the static channel, but it exists in the fading channel for $p_{\text {suc }}$ is less than 1 (Figure 4(a)). In the case of conservative CST (Figure 3(b)), when $x_{I} / x_{R}$ increases from -3 to -1 , the exposed terminal problem in the fading channel is less severe than that in the static channel because both $p_{\text {busy }}$ and $p_{\text {suc }}$ are less than 1 (Figure 4(b)). The hidden terminal problem does not exist in the static channel when $x_{I} / x_{R}$ is between 1 and 7 , but it occurs in the fading channel. To sum up, the random nature of fading depresses the probability of any dominating state.

We conclude that fading can mitigate severe hidden and exposed terminal problems due to its random nature.

Finally, we discuss the impact of communication parameters. Increasing $m$ makes a fading channel more like a static channel, resulting in sharper state transitions. Increasing packet size makes little change on state transitions because $p_{\text {idle }}$ is not affected by packet length and $p_{\text {suc }}$ barely changes. Increasing path-loss exponent makes signal power decay more severely, and therefore it shrinks the curves to the origin of $x_{I} / x_{R}=0$ and makes state transitions more sharply. We ignore the figures for space limitation.

4.2.2. Average Sensing Accuracy. How is the average sensing accuracy in fading channels compared with that in the static channel? To shed light on it, we perform the following simulation. We calculate average sensing accuracy for different CSTs when $\alpha$ is 3 on the topology of Figure 1(a). Here, $d_{T R}$ is fixed to be $10 \mathrm{~m}, d_{I T}$ varies from 0 to $k_{\max } \cdot d_{T R}$ with the step size of 


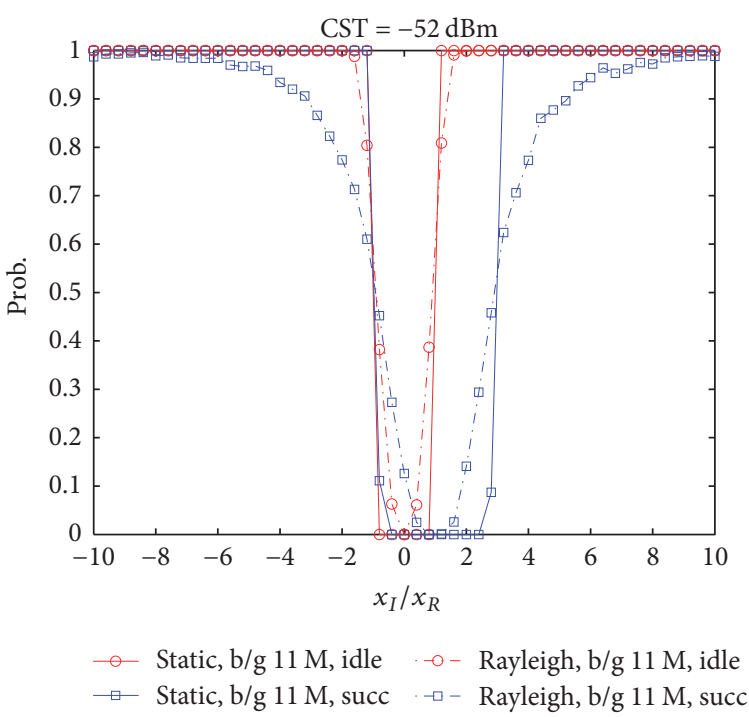

(a) Aggressive CST $=\bar{s}=-52 \mathrm{dBm}$

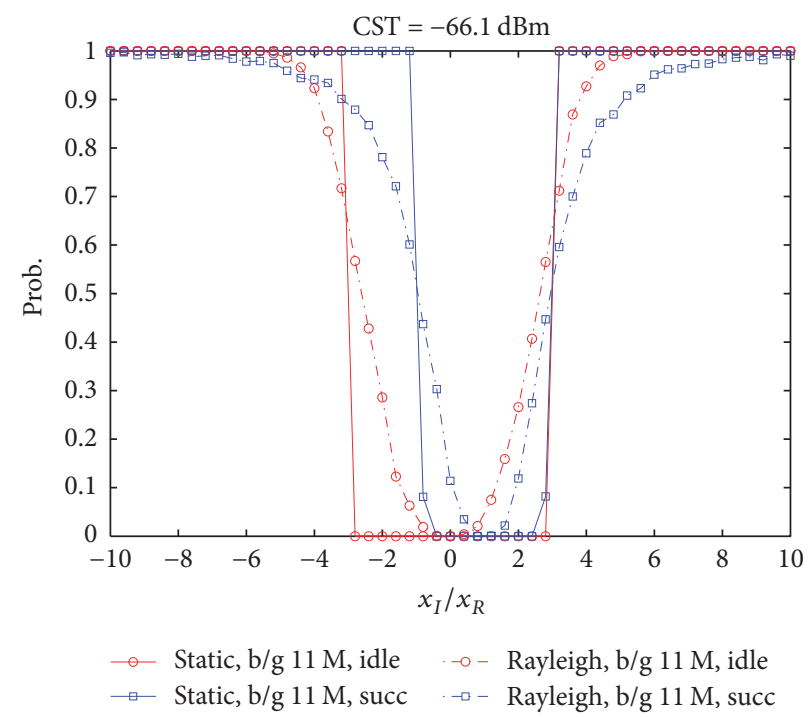

(b) Conservative $\operatorname{CST}=\bar{s} /\left(1+\beta_{0}^{1 / \alpha}\right)^{\alpha}=-66.1 \mathrm{dBm}$

FIGURE 4: Packet success ratio and channel idle probability of $802.11 \mathrm{~b} / \mathrm{g} 11 \mathrm{Mbps}$ as $x_{I} / x_{R}$ increases, comparing the static and Rayleigh channel ( $\alpha=3, x_{R}=10 \mathrm{~m}$, and packet size is 256 bytes).

$0.2 d_{T R}$, and $\theta$ varies from 0 to $2 \pi$ with the step size of $\pi / 12$, trying to cover all interferer locations. $k_{\max }$ reflects the area size. Figures 5(a)-5(c) show sensing accuracies of $802.11 \mathrm{~b} / \mathrm{g}$ $11 \mathrm{Mbps}$ for $k_{\max }=2,5,10$, respectively, and some interesting patterns are described as follows (we do not consider shadowing explicitly in the simulation, but one can find similar patterns because shadowing is like changing the propagation distance, which will be averaged out in this simulation).

First, fading tends to moderate the average sensing accuracy. For a given CST, fading degrades the probability of correct (or false) prediction states when they dominate, as said in Section 4.2.1.

Second, the average sensing accuracy of the static channel is higher than that of fading channels for optimally adjusted CSTs (about $-60 \mathrm{dBm}$ here), and their difference is more pronounced when the channel variation becomes larger. This result is verified for all bit rates for $m=0.5,1,2,5$ (see Appendix A.2), which shows that location asymmetry causes the suboptimality of sensing accuracy in the static channel for fixed carrier sensing thresholds, and the random nature of fading aggravates it further.

Third, we try to understand the trends of the curves in Figure 5. We use interference range in the static channel for a rough estimation of distance, which is $\beta_{0}^{1 / \alpha} \cdot d_{T R}=1.94 d_{T R}$ here. When $k_{\max }=2$ (Figure 5(a)) and the area is small, most interferer locations cause severe interference. And transmission should be avoided most of time and optimal CSTs prefer to be conservative. We can see that sensing accuracy is degraded by aggressive CSTs but insensitive to conservative CSTs. When $k_{\max }=5$ (Figure $5(\mathrm{~b})$ ) and the area is medium, some interferer locations result in significant hidden terminal problem and others result in significant exposed terminal problem. Therefore, sensing accuracy is sensitive to CSTs and the optimal one is $-60 \mathrm{dBm}$. When $k_{\max }=10$ (Figure 5(c)) and the area is large, most interferer locations cause slight interference. Consequently, aggressive CSTs are preferred to encourage transmissions.

\section{Range Scaling Laws in Nakagami Fading Channels}

Interference range and carrier sensing range are widely used in the static channel to visualize transmission states. We extend them to fading channels and derive their scaling laws.

\subsection{Interference Range}

$p_{\text {fail }}$-Interference Range $r_{I}\left(p_{\text {fail }}\right)$. It is defined to be the interferer-receiver radius (centered at the receiver) resulting in packet failure ratio equal to $p_{\text {fail }}$, and any interferer within the radius leads to larger packet failure ratio. The interference range is calculated under the single-interferer scenario based on the SNRT model ignoring thermal noise and receive sensitivity; nevertheless, it can provide a vivid view of interference level.

Recall the interference range $r_{I}^{\text {sta }}$ in the static channel:

$$
r_{I}^{\text {sta }}=\beta_{0}^{1 / \alpha} \cdot d_{T R}
$$

which is derived on the condition that $\bar{\beta}=\beta_{0}$. Any distance smaller (larger) than it leads to $p_{\text {fail }}=1\left(p_{\text {fail }}=0\right)$.

However, $p_{\text {fail }}$ is a continuous decreasing function $g(g$ can be derived from $F_{B}$, the cumulative distribution function of SIR) of the path-loss SIR in fading channels:

$$
p_{\text {fail }}=g(\bar{\beta})=g\left(\left(\frac{d_{I R}}{d_{T R}}\right)^{\alpha}\right) \text {. }
$$

So, the interference range $r_{I}^{\text {naka }}$ of the Nakagami fading model is a continuous monotonically decreasing function of $p_{\text {fail }}$ :

$$
r_{I}^{\text {naka }}\left(p_{\text {fail }}\right)=\left(g^{-1}\left(p_{\text {fail }}\right)\right)^{1 / \alpha} \cdot d_{T R}
$$




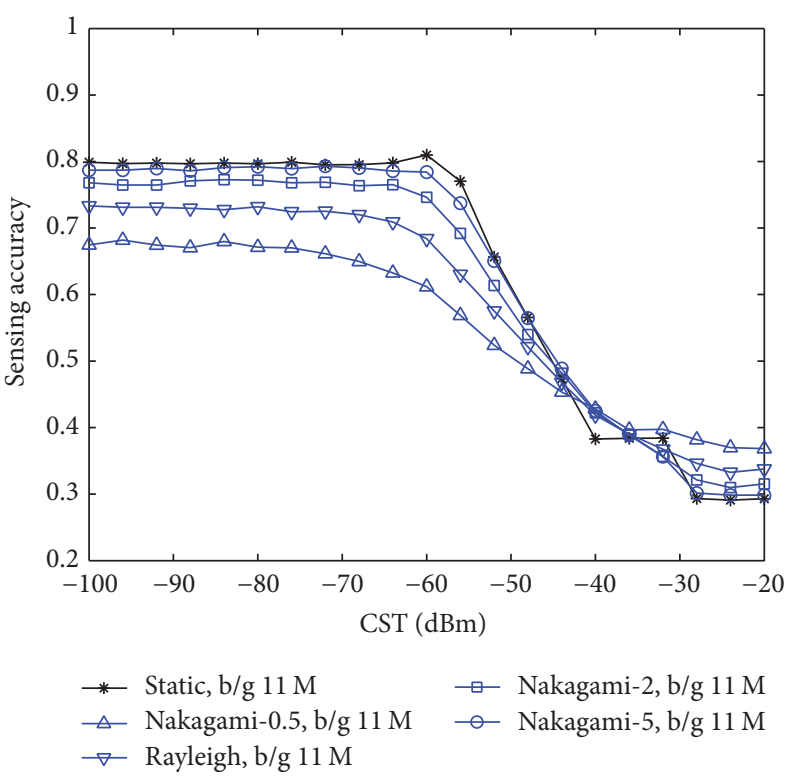

(a) $k_{\max }=2$

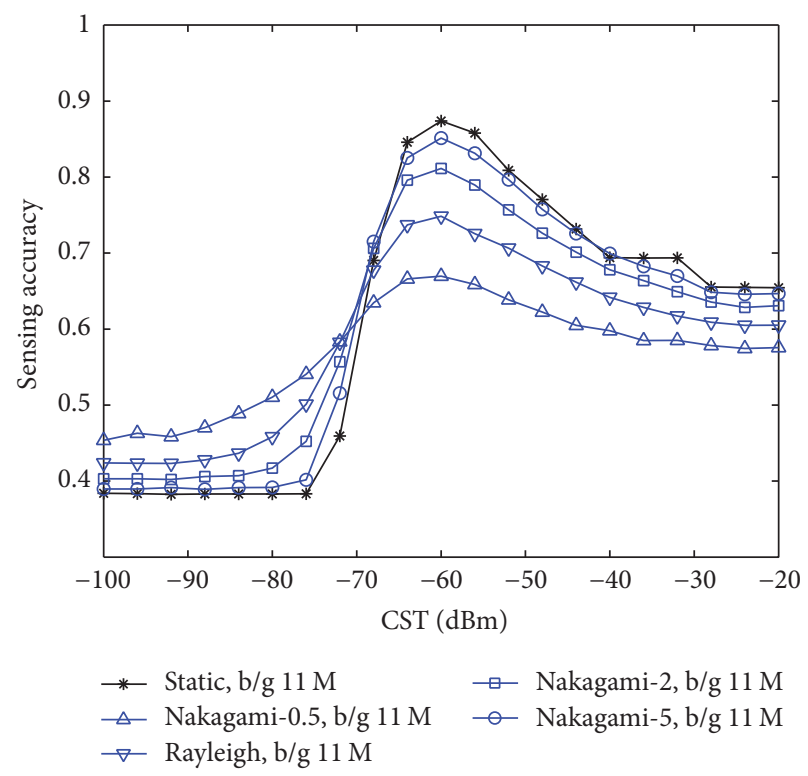

(b) $k_{\max }=5$

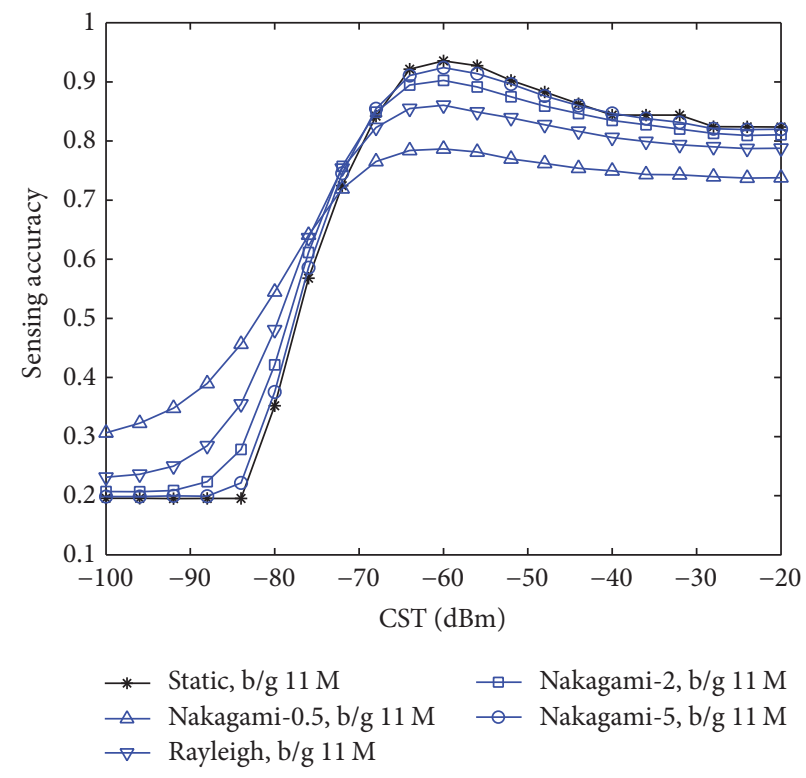

(c) $k_{\max }=10$

FIGURE 5: Average sensing accuracy as a function of carrier sensing threshold for $802.11 \mathrm{~b} / \mathrm{g} 11 \mathrm{Mbps}\left(\alpha=3, d_{T R}=10 \mathrm{~m}, \theta=\right.$ $0:(1 / 12) \pi:(11 / 12) \pi$, and packet size is 256 bytes $)$.

And the interference range of Rayleigh fading, $r_{I}^{\text {ray }}$, is

$$
r_{I}^{\text {ray }}\left(p_{\text {fail }}\right)=\left(\frac{1-p_{\text {fail }}}{p_{\text {fail }}}\right)^{1 / \alpha} \cdot r_{I}^{\text {sta }} .
$$

Figure 6 shows the $p_{\text {fail }}$-interference ranges in $802.11 \mathrm{~b} / \mathrm{g}$ $11 \mathrm{Mbps}$. One can read them off from the figure. For example, $r_{I}^{\text {ray }}(0.2)=3.08 d_{T R}$, larger than $r_{I}^{\text {sta }}=1.94 d_{T R}$. We are interested in interference ranges of small $p_{\text {fail }}$ 's in AP site planning. And smaller $m$ leads to larger interference ranges for the same $p_{\text {fail }}<0.5 . r_{I}^{\text {naka }}(0.5)$ is always equal to $r_{I}^{\text {sta }}$ (immediately by Lemma 2).
Before we prove the properties of $p_{\text {fail }}$-interference range, we first show the following lemma.

Lemma 5. The distribution of SIR $B$ in a Nakagami fading channel only depends on the path-loss $\operatorname{SIR} \bar{\beta}=\bar{s} / \bar{i}$, invariant of the scaling of $\bar{s}$ and $\bar{i}$.

Proof. See Appendix A.3.

Theorem 6. Scaling law of $p_{\text {fail }}$-interference range under Nakagami fading is as follows:

(1) The $p_{\text {fail }}$-interference range does not change with transmission power. 


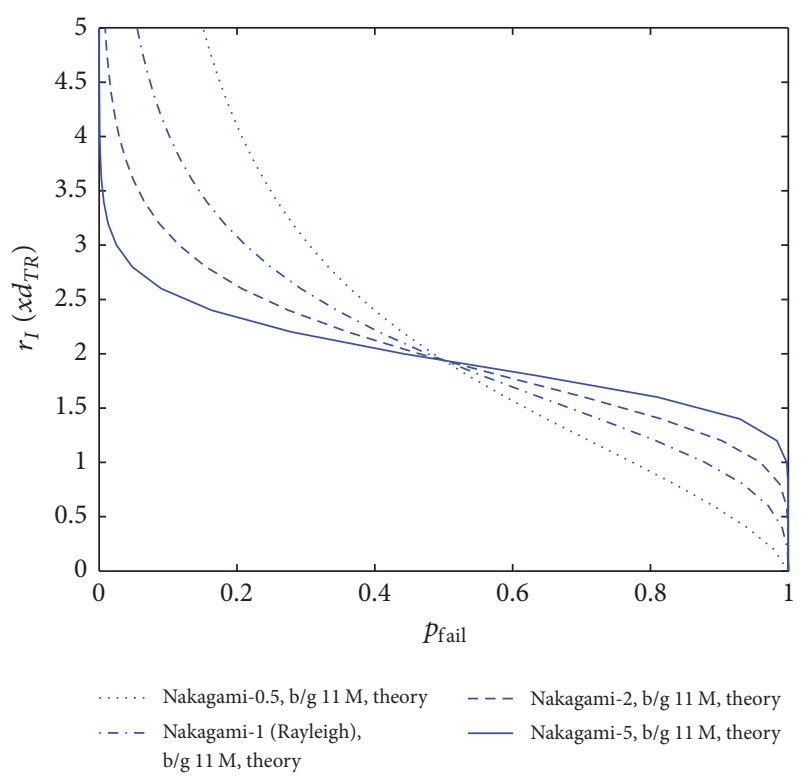

FIGURE 6: $p_{\text {fail }}$-interference range of $802.11 \mathrm{~b} / \mathrm{g} 11 \mathrm{Mbps}$ in the Nakagami fading model ( $\alpha=3$ and packet size is 256 bytes).

(2) The $p_{\text {fail }}$-interference range is in the form of $a_{1}^{1 / \alpha} \cdot d_{T R}$, where $a_{1} \triangleq a_{1}\left(\beta_{0}, p_{\text {fail }}\right)$ is independent of transmission power and the path-loss exponent $\alpha$.

Proof. (1) When we fix the distance of interference range and scale transmission power, $\bar{\beta}=\bar{s} / \bar{i}$ does not change, and the distribution of the SIR $B$ does not change either by Lemma 5 . So, $p_{\text {fail }}$ does not change since it only depends on the SIR distribution.

(2) $a_{1}=g^{-1}\left(p_{\text {fail }}\right)$ by (13) and is independent of transmission power by (1), so it is independent of $\alpha$.

Remark 7. The two properties are just like the scaling law of interference range in the static channel.

\subsection{Carrier Sensing Range}

$p_{\text {busy }}$-Carrier Sensing Range $r_{C}\left(p_{b u s y}\right)$. It is defined as the interferer-transmitter radius (centered at the transmitter) resulting in the channel busy probability equal to $p_{\text {busy }}$. Any interferer location within the radius leads to larger channel busy probability. Note that it is calculated by the singleinterferer scenario and it depends on transmission power and CST.

The channel busy probability $p_{\text {busy }}$ is an increasing function $h$ ( $h$ can be derived from $F_{I}$, the cumulative distribution function of $I$ ) of the average carrier sensing power $\bar{c}$ :

$$
p_{\text {busy }}=h(\bar{c})=h\left(P_{0}\left(\frac{d_{0}}{d_{T I}}\right)^{\alpha}\right) \text {, }
$$

where $P_{0}$ is the interference power at the reference distance $d_{0}$.
Recall the carrier sensing range in the static channel:

$$
r_{C}^{\text {sta }}=\left(\frac{P_{0}}{c_{0}}\right)^{1 / \alpha} \cdot d_{0},
$$

which is derived on the condition that $\bar{c}=c_{0}$ (the CST), and any distance less (larger) than it leads to $p_{\text {busy }}=1\left(p_{\text {busy }}=0\right)$.

The carrier sensing range $r_{C}^{\text {naka }}$ in the Nakagami fading model is a continuous monotonically decreasing function of $p_{\text {busy: }}$

$$
r_{C}^{\text {naka }}\left(p_{\text {busy }}\right)=\left(\frac{P_{0}}{h^{-1}\left(p_{\text {busy }}\right)}\right)^{1 / \alpha} \cdot d_{0} .
$$

By $p_{\text {busy }}=e^{-c_{0} / \bar{c}}$, the carrier sensing range of Rayleigh fading is

$$
r_{C}^{\text {ray }}\left(p_{\text {busy }}\right)=\left(-\log p_{\text {busy }}\right)^{1 / \alpha} \cdot r_{C}^{\text {sta }}
$$

Figures $7(\mathrm{a})$ and $7(\mathrm{~b})$ show the $p_{\text {busy }}$-carrier sensing ranges in Nakagami fading channels for two $c_{0}$ 's corresponding to $r_{C}^{\text {stat }}=d_{T R}$ and $r_{C}^{\text {stat }}=2 d_{T R}$, respectively.

We note that (b)'s $r_{C}^{\text {naka }}$ is exactly twice as (a)'s for the same $p_{\text {busy }}$, which is proved by the following theorem.

Theorem 8. Scaling law of $p_{\text {busy }}$-carrier sensing range under Nakagami fading is as follows:

(1) $p_{\text {busy }}$-carrier sensing range scales $1 / k^{1 / \alpha}$ time when the carrier sensing threshold scales $k$ time.

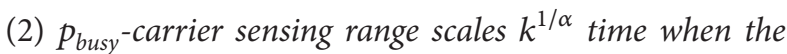
transmission power scales $k$ time.

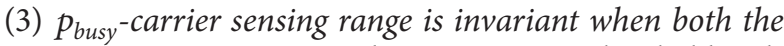
transmission power and carrier sensing threshold scale the same time.

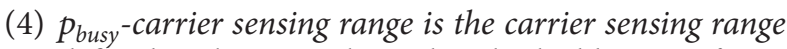
defined in the static channel multiplied by some factor $a_{2}{ }^{1 / \alpha}$, where $a_{2} \triangleq a_{2}\left(p_{\text {busy }}\right)$ is independent of transmission power, carrier sensing threshold, or the path-loss exponent $\alpha$.

Proof. (1) Let $r_{C}^{\text {naka }}\left(r_{C}^{\text {'naka }}\right)$ be $p_{\text {busy }}\left(p_{\text {busy }}^{\prime}\right)$-carrier sensing range for the CST $c_{0}\left(c_{0}^{\prime}\right)$. We prove the equivalent argument that $p_{\text {busy }}^{\prime}=p_{\text {busy }}$ when $c_{0}^{\prime}=k c_{0}$ and $r_{C}^{\text {naka }}=1 / k^{1 / \alpha} \cdot r_{C}^{\text {naka }}$.

Calculated by the path-loss model, $\bar{c}^{\prime}=k \bar{c}$ for $r_{C}^{\text {naka }}=$ $1 / k^{1 / \alpha} \cdot r_{C}^{\text {naka }}$.

$$
\begin{aligned}
p_{\text {busy }}^{\prime} & =\int_{c=k c_{0}}^{\infty} \frac{m^{m}}{\Gamma(m)(k \bar{c})^{m}} \cdot c^{m-1} e^{-m c /(k \bar{c})} d c \\
& =\int_{u=c_{0}}^{\infty} \frac{m^{m}}{\Gamma(m)(\bar{c})^{m}} \cdot u^{m-1} e^{-m u / \bar{c}} d u=p_{\text {busy }} .
\end{aligned}
$$

(2) Calculated by the path-loss model, $\bar{c}$ is unchanged when the transmission power scales $k$ times and the carrier sensing range scales $k^{1 / \alpha}$. So, $p_{\text {busy }}^{\prime}$ is equal to $p_{\text {busy }}$. 


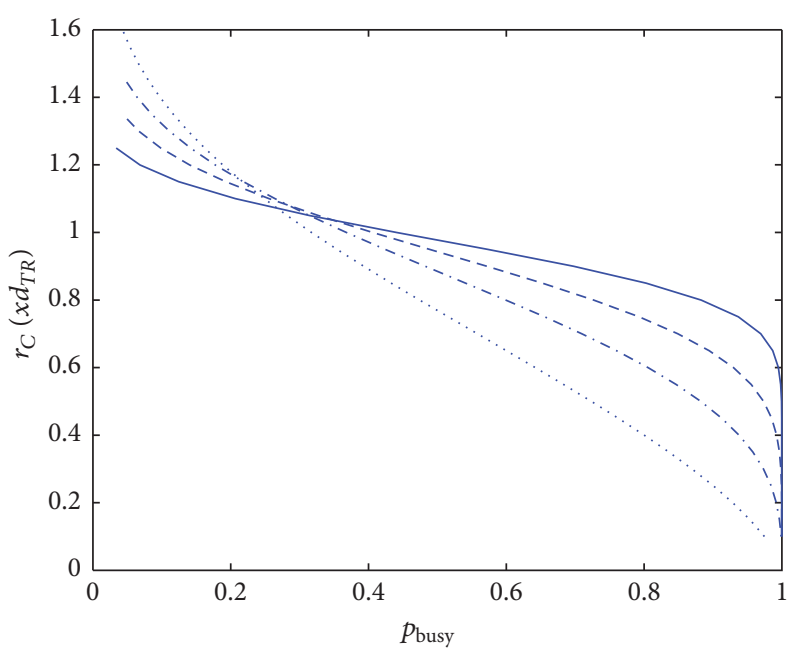

N... Nakagami-0.5, theory $\quad--$ Nakagami-2, theory
-.- Nakagami-1 (Rayleigh),
theory

(a) $r_{C}^{\text {stat }}=d_{T R}$

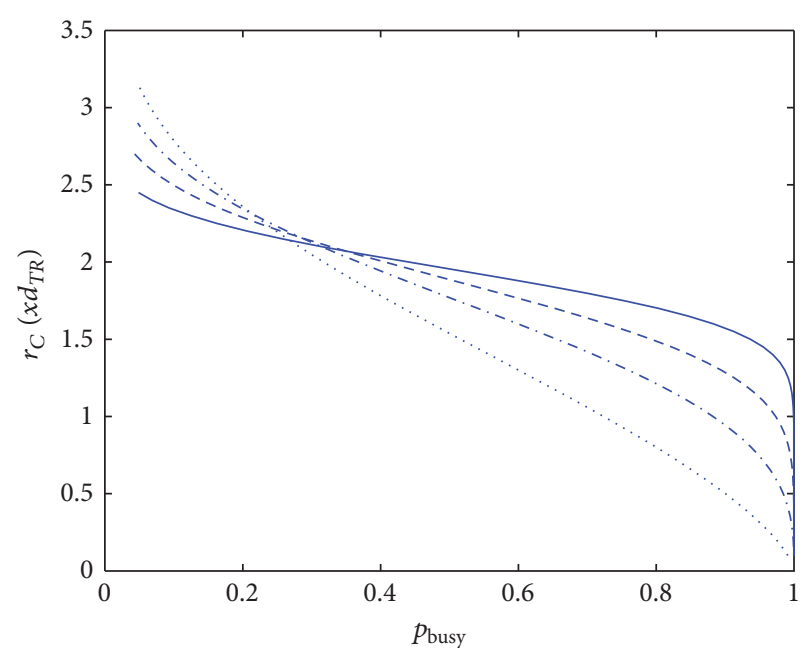

N... Nakagami-0.5, theory $\quad--$ Nakagami-2, theory
theory

(b) $r_{\mathrm{C}}^{\text {stat }}=2 d_{T R}$

FIGURE 7: $p_{\text {busy }}$-carrier sensing range in the Nakagami fading channels ( $\alpha=3$ and packet size is 256 bytes).

(3) By applying (1) and (2) subsequently, this argument immediately follows.

(4) From (16)-(17),

$$
r_{C}^{\text {naka }}\left(p_{\text {busy }}\right)=\left(\frac{c_{0}}{h^{-1}\left(p_{\text {busy }}\right)}\right)^{1 / \alpha} \cdot r_{C}^{\text {sta }} .
$$

Let $a_{2}=c_{0} / h^{-1}\left(p_{\text {busy }}\right)$. By the properties (1) and (2), both $r_{C}^{\text {naka }}\left(p_{\text {busy }}\right)$ and $r_{C}^{\text {sta }}$ scale the same time when transmission power or CST scales, so $a_{2}$ is independent of them. Because $\alpha$ is not a parameter of $h^{-1}, a_{2}$ is independent of $\alpha$.

Remark 9. The first three properties are just like the scaling law in the static channel. The fourth one reveals the simple relationship between carrier sensing range in the Nakagami fading channel and that in the static channel.

5.3. Implications. First, interference range and carrier sensing range help us to visualize transmission states in fading channels. Figure 8 plots them for a link (arrowed line). We can see $p_{\text {fail }}$ and $p_{\text {busy }}$ of any location and examine their consistency. For example, as an interferer is moving along the 0.9 -interference range (the inner most solid circle around the receiver) and is away from the transmitter, it crosses the $0.9,0.7,0.5$-carrier sensing ranges (the three inner dashed circles around the transmitter) in sequence. As a result, the hidden terminal problem becomes severer $\left(p_{\text {idle }} p_{\text {fail }}=\right.$ $0.09,0.27,0.45$, resp.). Taking another example, we compare the two cross dots in the figure where $\left(p_{\text {idle }}, p_{\text {fail }}\right)=(0.5,0.9)$ and $(0.9,0.5)$, respectively. Indicated by this example, the hidden terminal problem may not be mitigated as the interferer is moving away from the receiver, for it is more invisible in the transmitter's detection (i.e., $p_{\text {busy }}$ is decreasing).

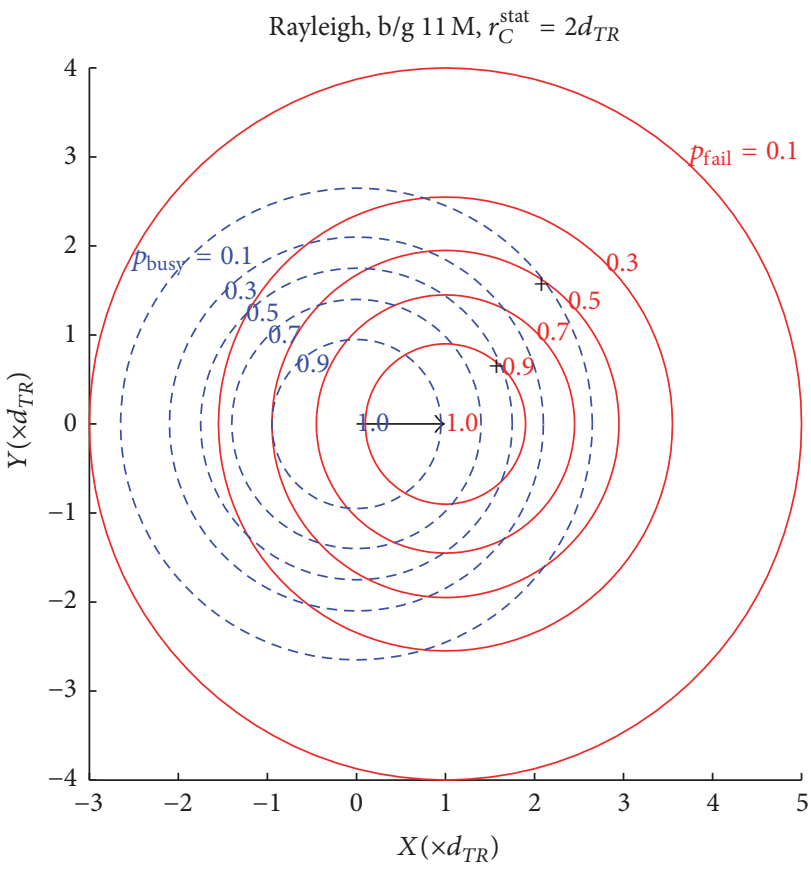

FIGURE 8: Some $p_{\text {fail }}$-interference ranges (for $802.11 \mathrm{~b} / \mathrm{g} 11 \mathrm{Mbps}$ ) and $p_{\text {busy }}$-carrier sensing ranges $\left(r_{C}^{\text {stat }}=2 d_{T R}\right)$ in the Nakagami-1 (Rayleigh) fading channel ( $\alpha=3$ and packet size is 256 bytes).

Second, we extend interference and carrier sensing regions in the static channel to fading channels. In Figure 9, $A_{C}$ denotes the region within the carrier sensing range and $A_{I}$ denotes the region within the interference range. In the static channel (Figure 9(a)), $A_{C}-A_{I}, A_{C} \cap A_{I}, A_{I}-A_{C}$, and $\overline{A_{I}} \cup \overline{A_{C}}$ are the regions of (busy, suc), (busy, fail), (idle, 


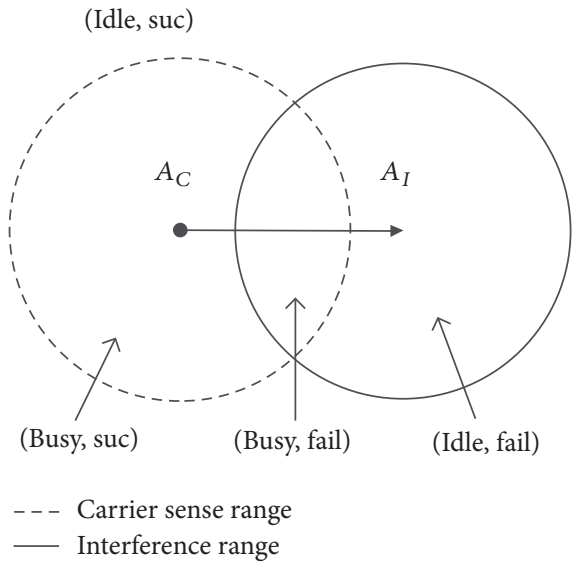

(a) Static channel

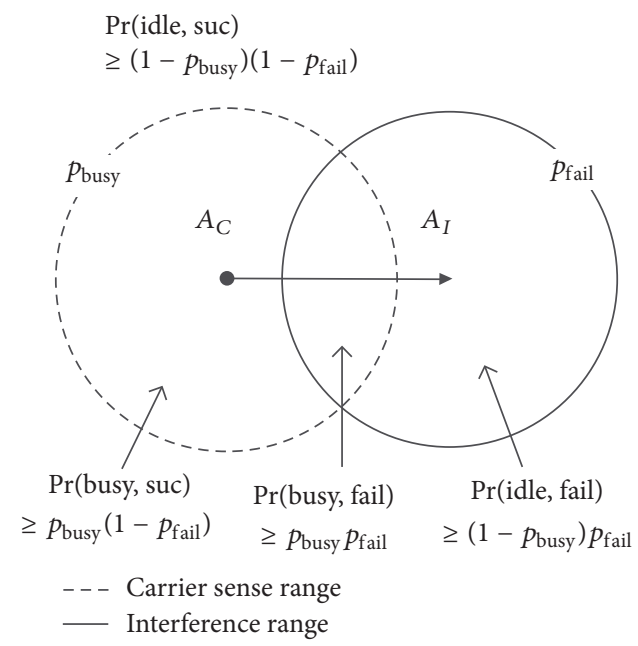

(b) Fading channel

FIGURE 9: Regions of interference and carrier sensing ranges, comparing the static and fading channels.

fail), and (idle, suc), respectively. In contrast, in the fading channel case (Figure 9(b)), the four regions divided by $p_{\text {busy }}{ }^{-}$ carrier sensing range and $p_{\text {idle }}$-interference range give lowerbound probabilities of the counterpart states. For example, the probability of (busy, suc) for any interferer within $A_{C}-A_{I}$ is larger than or equal to $p_{\text {busy }}\left(1-p_{\text {fail }}\right)$.

Finally, the scaling theorems suggest that it is unnecessary to exhaustively simulate all different topologically relevant parameters when verifying some empirical rule; instead, using one topology is enough. Here, topologically relevant parameters are link distance, path-loss exponent, shadowing factor (the shadowing factor is like stretching or shrinking the link distance, so we can attribute it to the case of link distance), carrier sensing threshold, and transmission power, which are related to interference range or carrier sensing range as well as the accompanying probabilities of $p_{\text {fail }}$ or $p_{\text {busy }}$. We illustrate this application by the following examples. In Figures 3-4, we fix CST and transmission power to some values in the simulation, and the curves are of similar shapes when they change (by Theorem 8). In Figure 5, we can get similar curves by changing $k_{\max }$ accordingly with the changing of some topologically relevant parameter. So, we are confident that our observation findings are of generality.

\section{Conclusion}

In this work, we reexamine the probabilistic effects of multipath fading on interference and carrier sensing from a micro-view of a two-link topology. We find that fading is not always bad; sometimes it helps. For example, fading can let traffic go through the channel under high interference (unlike the static channel), and fading can mitigate severe hidden or exposed terminal problems. Theoretically, we prove the condition of optimal channel accessing probability just like the static channel. And we define the interference range and carrier sensing range for fading channels and prove that their scaling laws are just like those in the static channel, with the application of making one simulation result confident without trying all different topologically relevant parameters. In the future, one extension is to examine our observations in large ad hoc networks and model them by stochastic geometry.

\section{Appendix}

\section{A. Supportive Proofs and Simulation Results}

\section{A.1. Proof of Lemma 2}

Proof. At $\bar{\beta}=\beta_{0}$, ignoring $s_{0}$,

$$
p_{\text {suc }}=1-F_{B}\left(\beta_{0}\right)=\int_{s=0}^{\infty} f_{S}(s) F_{I}\left(\frac{s}{\beta_{0}}\right) d s,
$$

where $F_{X}\left(f_{X}\right)$ is defined to be the cumulative distribution function (probability density function) of the random variable $X$.

From $f_{I}(i)=F_{I}^{\prime}(i)$,

$$
\frac{d F_{I}\left(s / \beta_{0}\right)}{d s}=f_{I}\left(\frac{s}{\beta_{0}}\right) \cdot \frac{1}{\beta_{0}} .
$$

Integrating it on both sides, we get

$$
F_{I}\left(\frac{s}{\beta_{0}}\right)=\int_{0}^{s} f_{I}\left(\frac{s}{\beta_{0}}\right) \cdot \frac{1}{\beta_{0}} d s .
$$

In the Nakagami fading model, $S$ and $I$ follow the Gamma distribution (2):

$$
\begin{aligned}
& f_{S}(s)=\frac{m^{m}}{\Gamma(m) \bar{s}^{m}} \cdot s^{m-1} e^{-m s / \bar{s}}, \\
& f_{I}(i)=\frac{m^{m}}{\Gamma(m) \bar{i}^{-m}} \cdot i^{m-1} e^{-m i / \bar{i}} .
\end{aligned}
$$



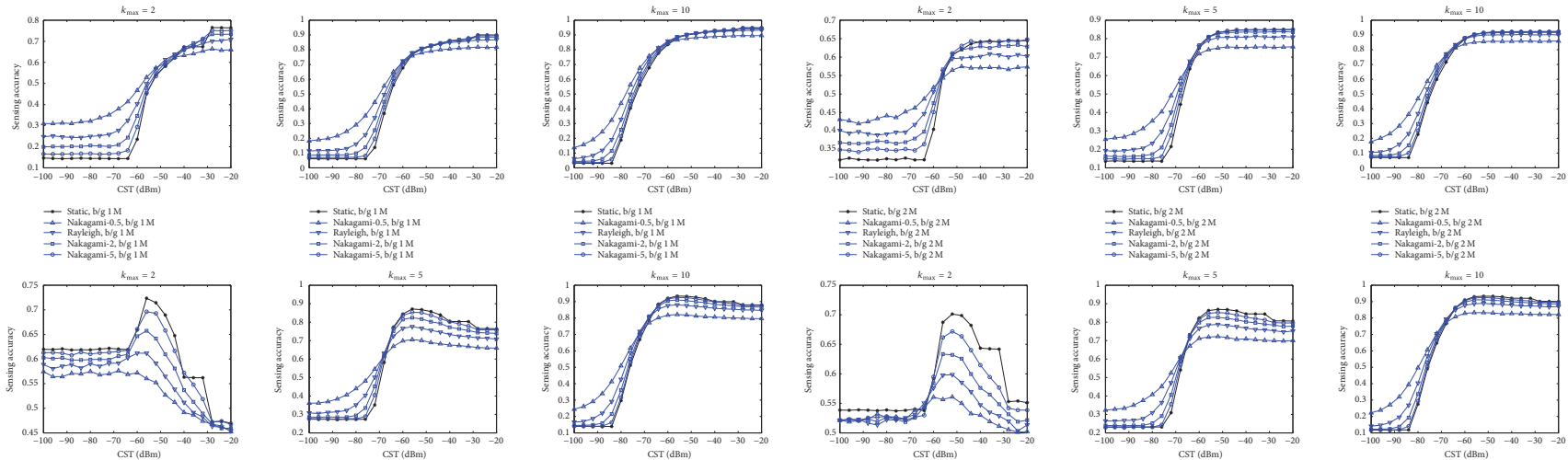

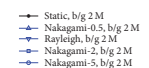

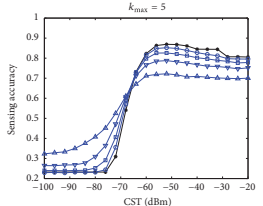

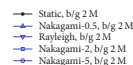

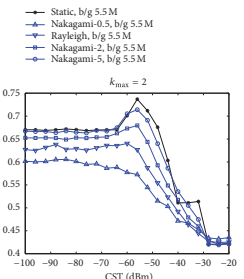

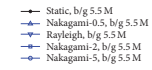

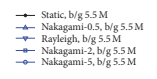
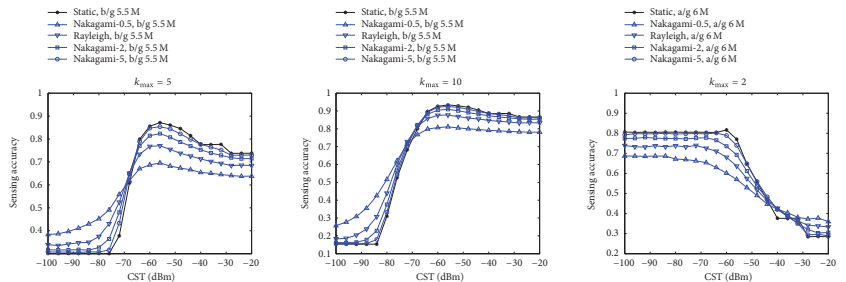

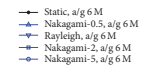
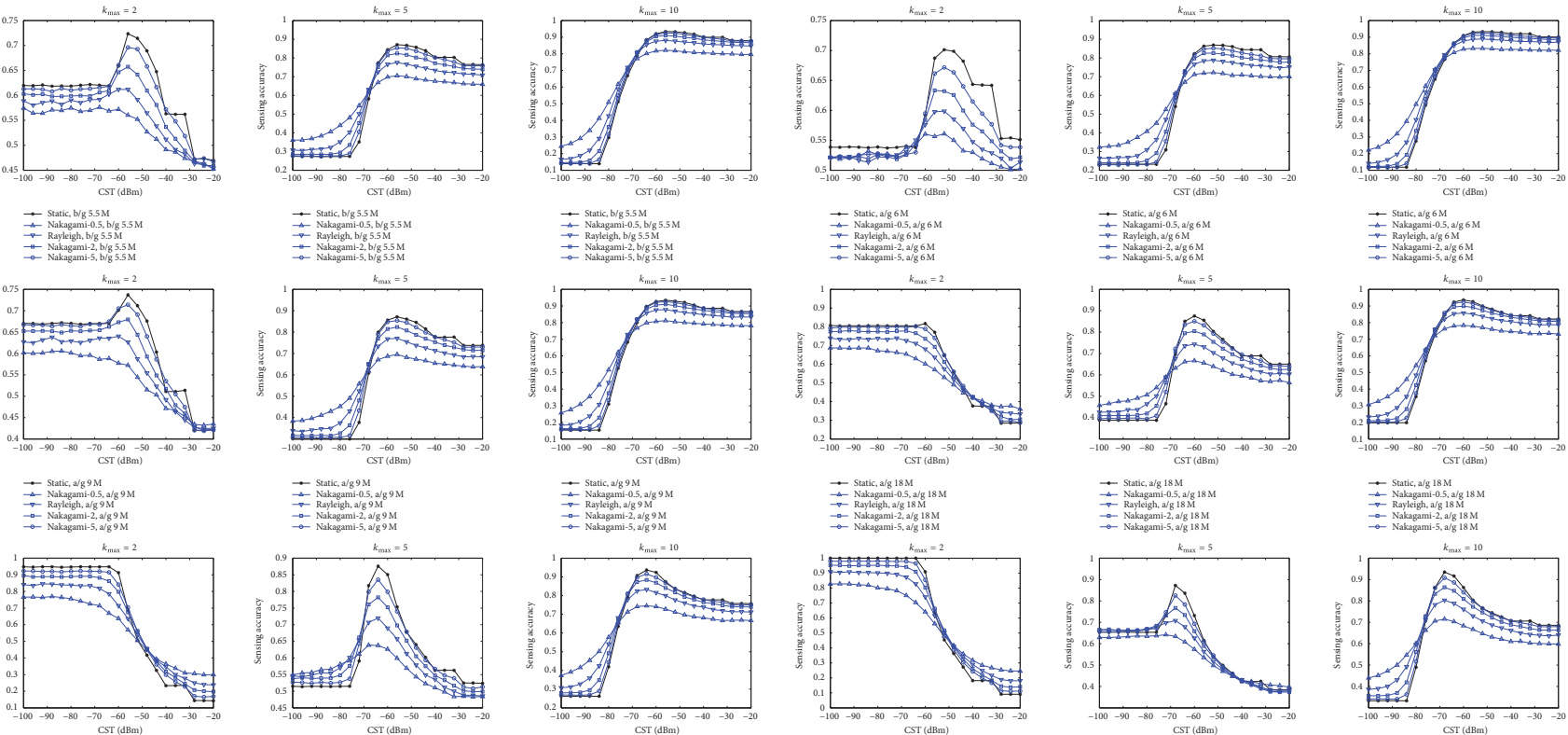

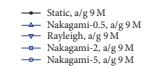

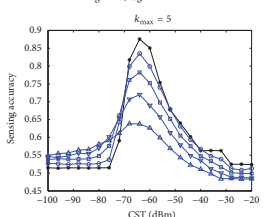

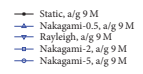
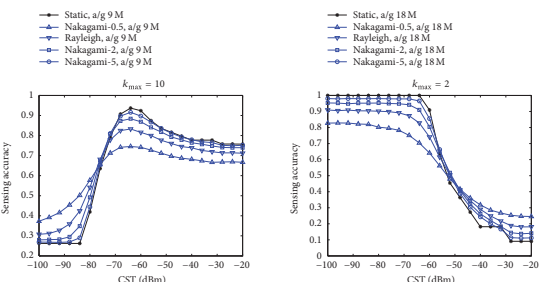

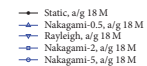

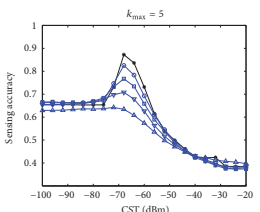

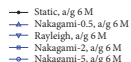
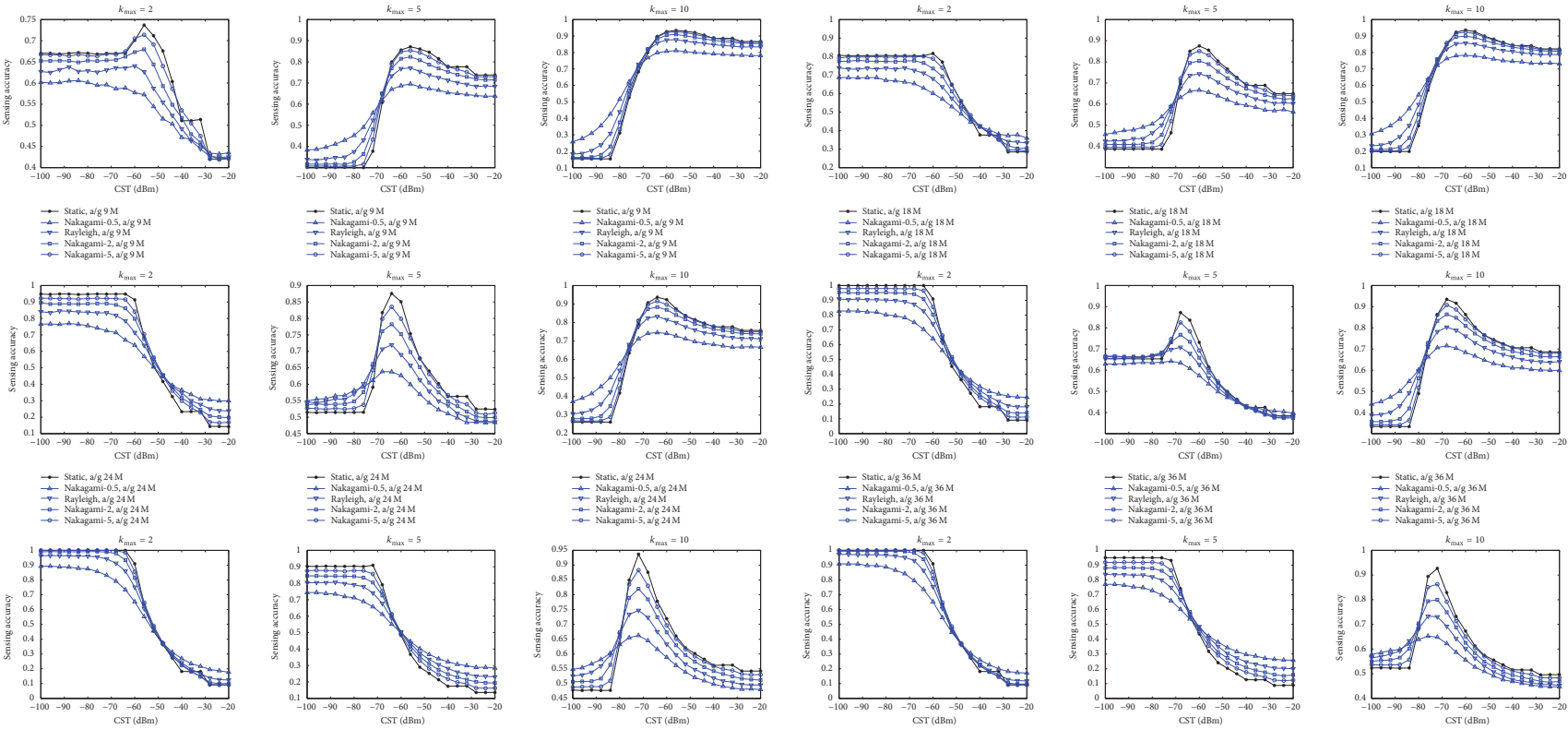

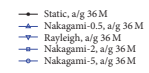

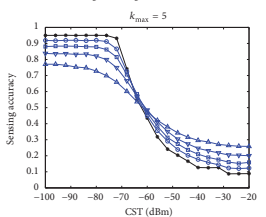

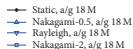
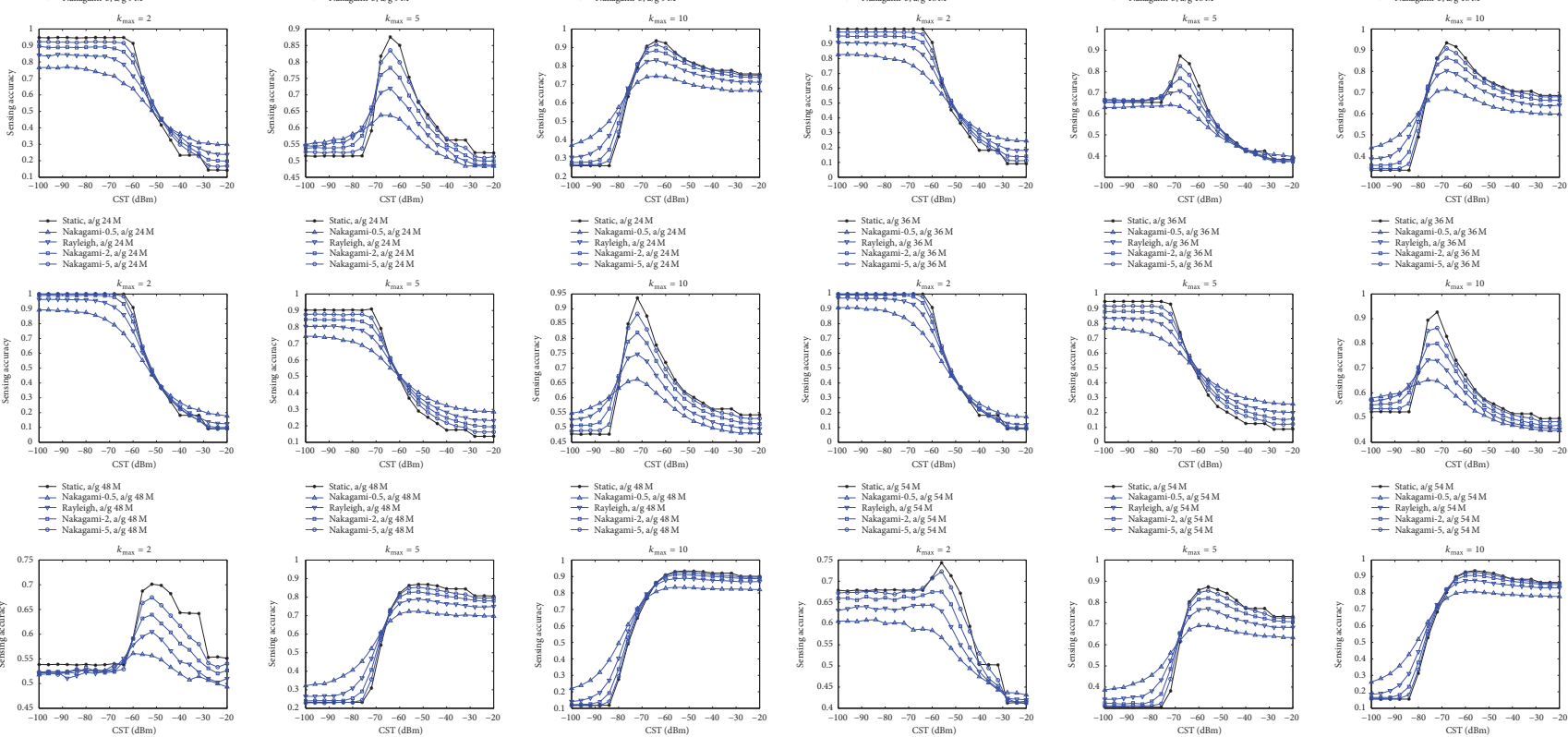

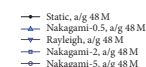

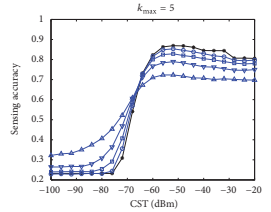

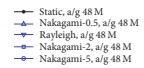
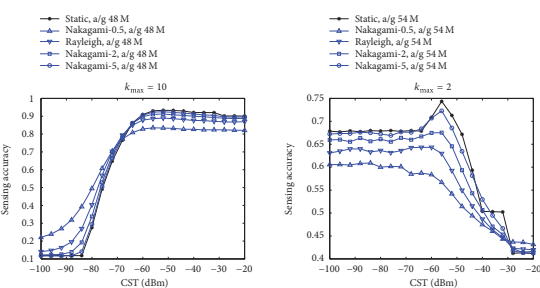

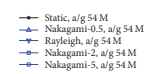

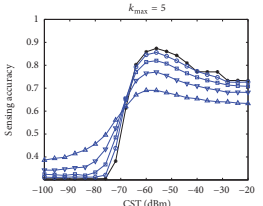

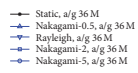
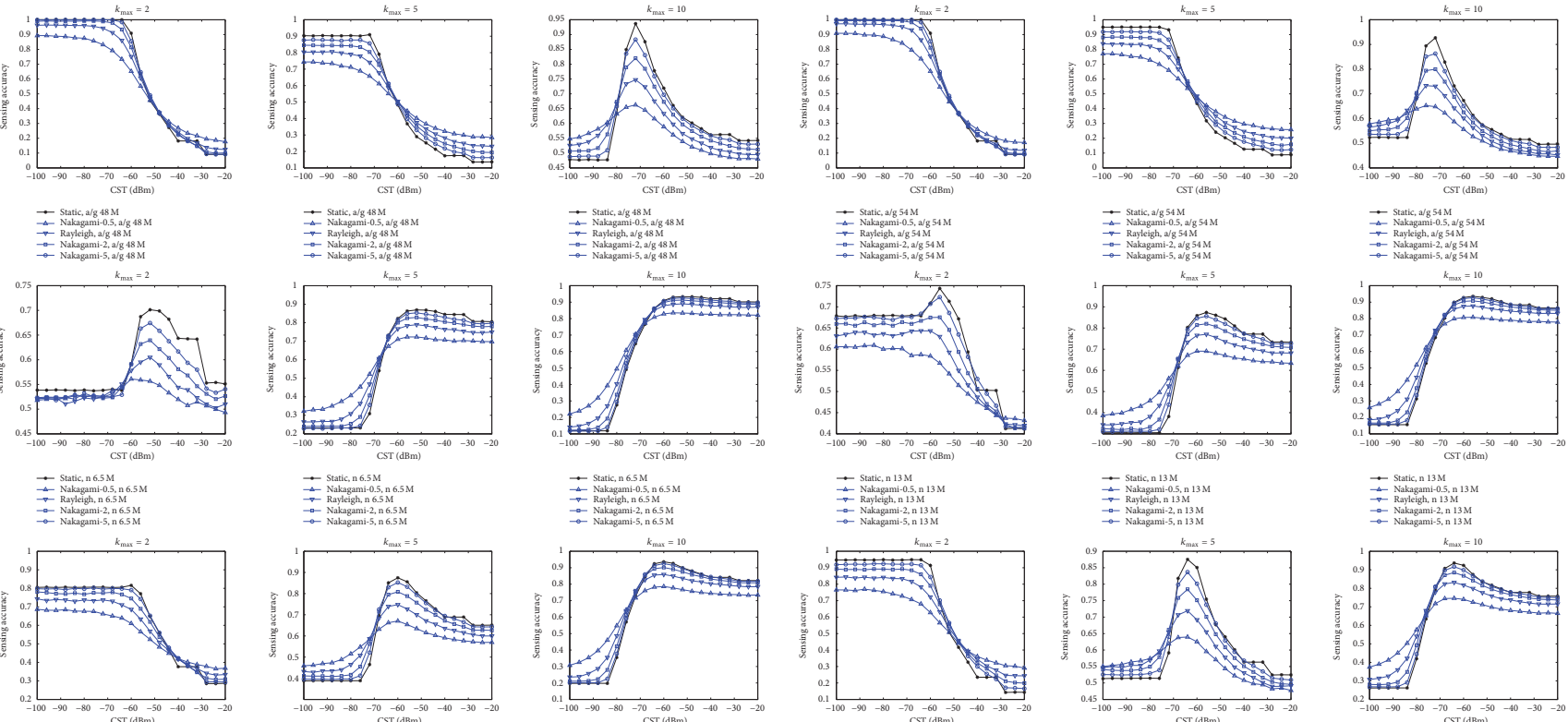

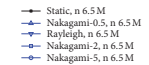

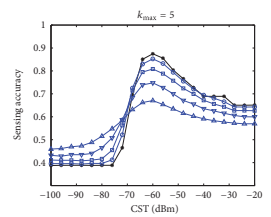

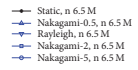
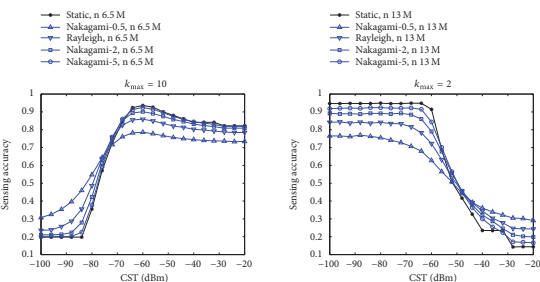

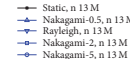

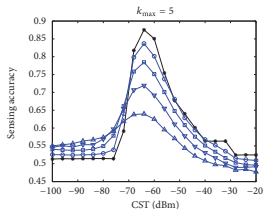

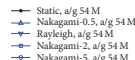
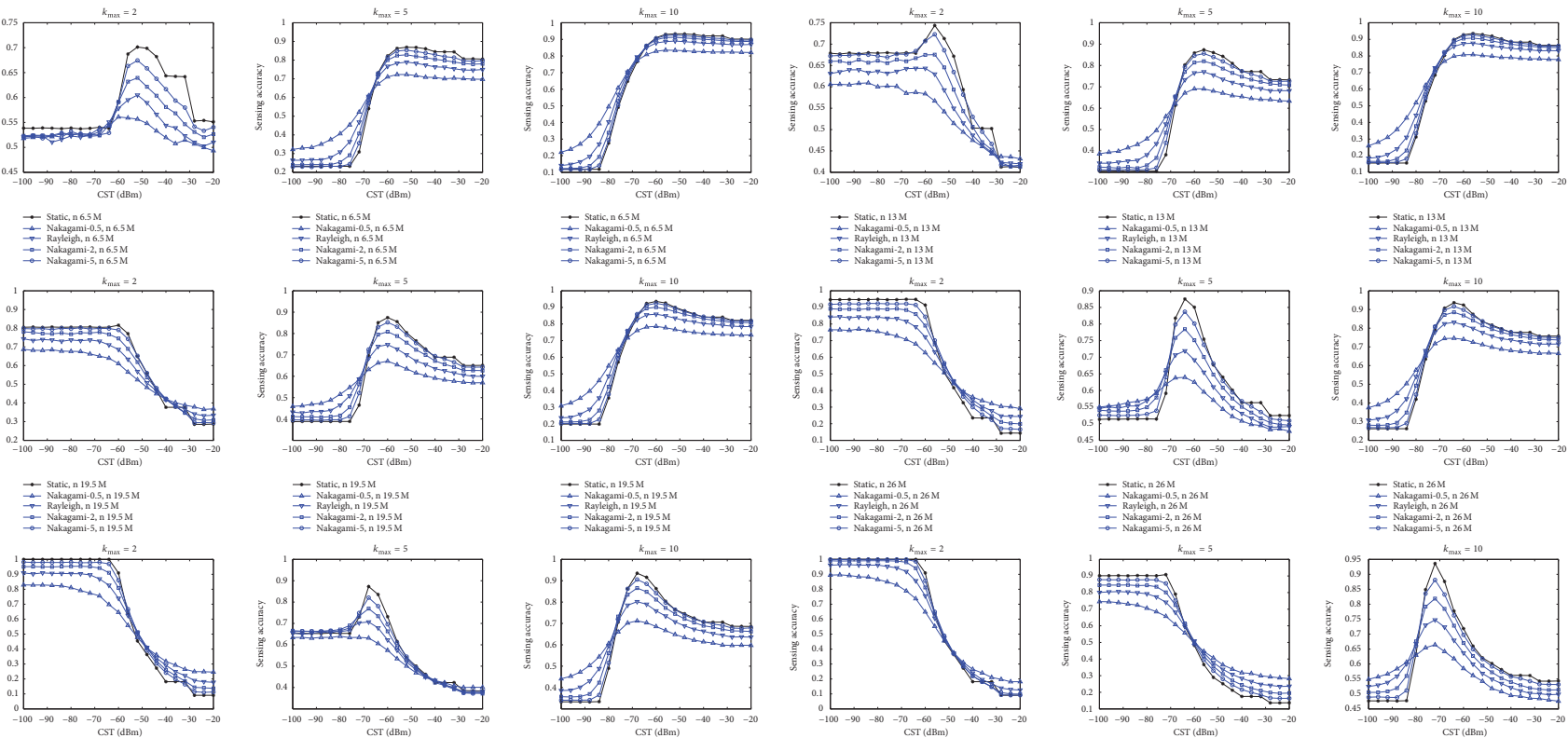

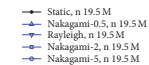

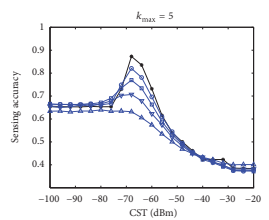

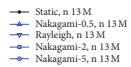

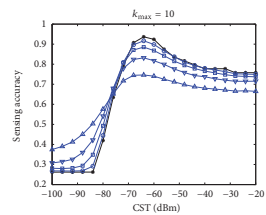

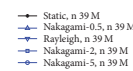

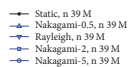

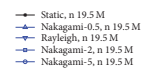

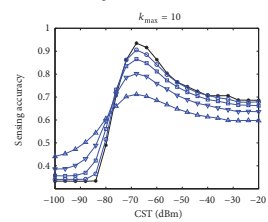

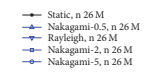

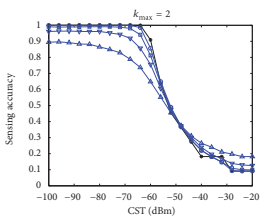

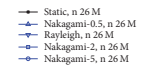

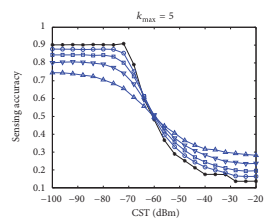

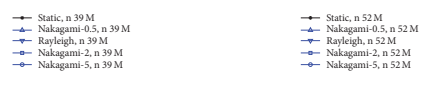

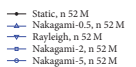

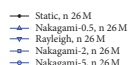

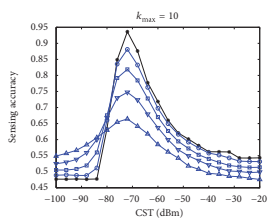

FIgUre 10: Continued. 


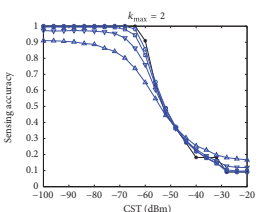

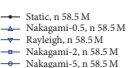
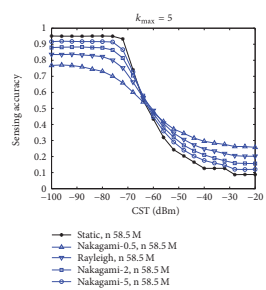

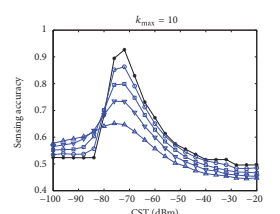

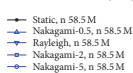

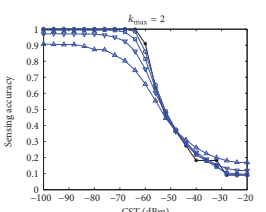

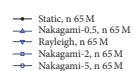
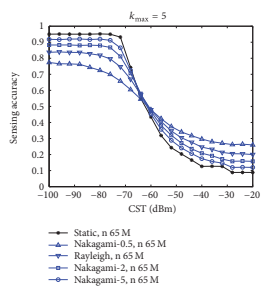

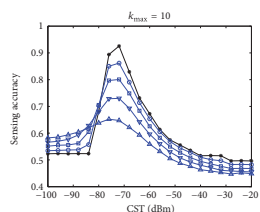

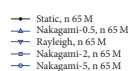

FIGURE 10: Carrier sensing accuracies for the other bit rates of $802.11 \mathrm{a} / \mathrm{b} / \mathrm{g} / \mathrm{n}\left(k_{\max }=2,5,10\right)$.

Combining (A.3) and (A.5) under the condition that $\bar{\beta}=$ $\bar{s} / \bar{i}=\beta_{0}$, one can find that

$$
F_{I}\left(\frac{s}{\beta_{0}}\right)=F_{S}(s) .
$$

So, (A.1) turns into a simple form:

$$
p_{\text {suc }}=\int_{F_{S}(s)=0}^{1} F_{S}(s) d F_{S}(s)=0.5 \text {. }
$$

Note that this property is independent of $m$.

A.2. Carrier Sensing Accuracies for the Other Bit Rates. See Figure 10.

\section{A.3. Proof of Lemma 5}

Proof. Suppose we have two sets of signal and interference powers: $(S, I)$ and $\left(S^{\prime}, I^{\prime}\right)$. The expectation of the latter scales $k$ time that of the former; that is, $\overline{s^{\prime}}=k \bar{s}$ and $\overline{i^{\prime}}=k \bar{i}$.

From (2),

$$
\begin{aligned}
& f_{S^{\prime}}(s)=\frac{m^{m}}{\Gamma(m)(k \bar{s})^{m}} \cdot s^{m-1} e^{-m s /(k \bar{s})}, \\
& f_{I^{\prime}}(i)=\frac{m^{m}}{\Gamma(m)(k \bar{i})^{m}} \cdot i^{m-1} e^{-m i /(k \bar{i})} .
\end{aligned}
$$

The pdf of $B^{\prime}=S^{\prime} / I^{\prime}$ is

$$
\begin{aligned}
f_{B^{\prime}}(\beta) & =\int_{s=0}^{\infty} \frac{f_{S^{\prime}}(s) f_{I^{\prime}}(s / \beta) s}{\beta^{2} d s} \\
& =\int_{u=0}^{\infty} \frac{f_{S}(u) f_{I}(u / \beta) u}{\beta^{2} d u}=f_{B}(\beta),
\end{aligned}
$$

where we use the substitution of $u=k s$ to show that it is equal to the pdf of $B=S / I$.

So, the distribution of SIR is invariant of the power scaling.

\section{Symbols}

S: $\quad$ Random variable of signal power

I: $\quad$ Random variable of interference power

B: $\quad$ Random variable of SIR (in ratio)

$s_{0, v}: \quad$ Receive sensitivity for bit rate $v$

$c_{0, v}: \quad$ Carrier sense threshold for bit rate $v$

$\beta_{0, v}: \quad$ SNR threshold for bit rate $v$ in the SNRT model

$n_{0}$ : $\quad$ Constant thermal noise, $-95.6 \mathrm{dBm}$

$\bar{\beta}$ : $\quad$ Path-loss SIR, defined by the average signal power over the average interference power

$p_{\text {suc, } v}(\beta)$ : Packet success ratio for bit rate $v$ and SNR $\beta$

$p_{\text {suc }, v}: \quad$ Expectation of packet success ratio for bit rate $v$

$p_{\text {idle }}$ Channel idle probability.

\section{Conflicts of Interest}

The authors declare that there are no conflicts of interest regarding the publication of this paper.

\section{Acknowledgments}

This work is supported by the National Natural Science Foundation of China (no. 61309030, no. 61502315), the Joint Funds of the National Natural Science Foundation of China (U1509214), and the Foundation Research Project of Beijing University of Technology in 2015 (025000514315502) and granted by Discipline Construction Foundation of Central University of Finance and Economics as well as Young Teacher Development Fund of Central University of Finance and Economics (QJJ1706).

\section{References}

[1] K. Xu, M. Gerla, and S. Bae, "How effective is the IEEE 802.11 RTS/CTS handshake in ad hoc networks?" in Proceedings of the IEEE Global Telecommunications Conference (GLOBECOM '02), vol. 1, pp. 72-76, November 2002.

[2] M. Z. Brodsky and R. T. Morris, "In defense of wireless carrier sense," in Proceedings of the ACM SIGCOMM 2009 Conference on Data Communication, SIGCOMM 2009, pp. 147-158, August 2009.

[3] F. Hansen and F. I. Meno, "Mobile fading Rayleigh and lognormal superimposed," IEEE Transactions on Vehicular Technology, vol. 26, no. 4, pp. 332-335, 1977. 
[4] K. W. Sowerby and A. G. Williamson, "Outage probability calculations for a mobile radio system having multiple rayleigh inteferers," Electronics Letters, vol. 23, no. 11, pp. 600-601, 1987.

[5] Y.-D. Yao and A. U. H. Sheikh, "Outage probability analysis for microcell mobile radio systems with cochannel interferers n Rician/Rayleigh fading environment," Electronics Letters, vol. 26, no. 13, pp. 864-866, 1990.

[6] J. H. Kim and J. K. Lee, "Capture effects of wireless CSMA/CA protocols in Rayleigh and shadow fading channels," IEEE Transactions on Vehicular Technology, vol. 48, no. 4, pp. 12771286, 1999.

[7] C. Song, H. R. Sharif, and P. Mahasukhon, "Evaluating saturation throughput performance of the IEEE 802.11 MAC under fading channels," in Proceedings of the 2nd International Conference on Broadband Networks, BROADNETS 2005, pp. 726-731, October 2005.

[8] F. Daneshgaran, M. Laddomada, F. Mesiti, and M. Mondin, "Unsaturated throughput analysis of IEEE 802.11 in presence of non ideal transmission channel and capture effects," IEEE Transactions on Wireless Communications, vol. 7, no. 4, pp. 1276 1286, 2008.

[9] E. J. Leonardo and M. D. Yacoub, "Exact formulations for the throughput of IEEE 802.11 DCF in Hoyt, Rice, and Nakagami$\mathrm{m}$ fading channels," IEEE Transactions on Wireless Communications, vol. 12, no. 5, pp. 2261-2271, 2013.

[10] J. Sheng and K. S. Vastola, "An efficient model of 802.11 ad hoc networks under a block-fading rayleigh channel with physical layer capture," in Proceedings of the 2008 IEEE Global Telecommunications Conference, GLOBECOM 2008, pp. 656660, December 2008.

[11] H. Chang, V. Misra, and D. Rubenstein, "A general model and analysis of physical layer capture in 802.11 networks," in Proceedings of the INFOCOM 2006: 25th IEEE International Conference on Computer Communications, April 2006.

[12] S. G. Glisic, R. Rao, and L. B. Milstein, "The effect of imperfect carrier sensing on nonpersistent carrier sense multiple access," in Proceedings of the IEEE International Conference on Communications - ICC '90 Part 3 (of 4), pp. 1266-1269, April 1990.

[13] S. G. Glisic, "1-persistent carrier sense multiple access in radio channels with imperfect carrier sensing," IEEE Transactions on Communications, vol. 39, no. 3, pp. 458-464, 1991.

[14] I. Ramachandran and S. Roy, "Analysis of throughput and energy efficiency of p-persistent CSMA with imperfect carrier sensing," in Proceedings of the GLOBECOM'05: IEEE Global Telecommunications Conference, 2005, pp. 3428-3432, December 2005.

[15] J. W. Chong, D. K. Sung, and Y. Sung, "Cross-layer performance analysis for CSMA/CA protocols: impact of imperfect sensing," IEEE Transactions on Vehicular Technology, vol. 59, no. 3, pp. 1100-1108, 2010.

[16] J. Sheng and K. S. Vastola, "Physical carrier sensing outage in single hop IEEE 802.11 ad hoc networks with slowly moving stations," in Proceedings of the IEEE Wireless Communications and Networking Conference, WCNC 2008, pp. 1350-1355, April 2008.

[17] C. H. Kai and S. C. Liew, "Towards a more accurate carrier sensing model for CSMA wireless networks," in Proceedings of the 2010 IEEE International Conference on Communications, ICC 2010, May 2010.

[18] J. Sheng and K. S. Vastola, "Performance modeling of 802.11 ad hoc networks with time-varying carrier sense range and physical capture capability," in Proceedings of the 2008 IEEE Global Telecommunications Conference, GLOBECOM 2008, pp. 325-329, December 2008.

[19] J. Dai and Y. Yamao, "Performance of CSMA/CA multihop network considering intra-flow interference under fading environment," in Proceedings of the 2013 IEEE 77th Vehicular Technology Conference, VTC Spring 2013, June 2013.

[20] J. Mittag and H. Hartenstein, "Is CSMA able to coordinate multiple access in vehicular radio channels effectively?" in Proceedings of the 2012 12th International Conference on ITS Telecommunications, ITST 2012, pp. 801-806, November 2012.

[21] H. Schumacher and H. Tchouankem, "Highway propagation modeling in VANETS and its impact on performance evaluation," in Proceedings of the 2013 10th Annual Conference on Wireless On-Demand Network Systems and Services, WONS 2013, pp. 178-185, March 2013.

[22] S. N. Chiu, D. Stoyan, W. S. Kendall, and J. Mecke, Stochastic Geometry and its Applications, John Wiley \& Sons, Hoboken, NJ, USA, 3rd edition, 2013.

[23] F. Baccelli, B. o. Blaszczyszyn, and P. Muhlethaler, "An aloha protocol for multihop mobile wireless networks," Institute of Electrical and Electronics Engineers. Transactions on Information Theory, vol. 52, no. 2, pp. 421-436, 2006.

[24] H. Q. Nguyen, F. Baccelli, and D. Kofman, "A stochastic geometry analysis of dense IEEE 802.11 networks," in Proceedings of the IEEE INFOCOM 2007: 26th IEEE International Conference on Computer Communications, pp. 1199-1207, May 2007.

[25] M. Haenggi, "A geometric interpretation of fading in wireless networks: theory and applications," Institute of Electrical and Electronics Engineers. Transactions on Information Theory, vol. 54, no. 12, pp. 5500-5510, 2008.

[26] M. Haenggi and R. K. Ganti, "Interference in Large Wireless Networks," Foundations and Trends in Networking, vol. 3, no. 2, pp. 127-248, 2009.

[27] M. Kaynia, G. E. Øien, and N. Jindal, "Impact of fading on the performance of ALOHA and CSMA," in Proceedings of the 2009 IEEE 10th Workshop on Signal Processing Advances in Wireless Communications, SPAWC 2009, pp. 394-398, June 2009.

[28] T. Yang, G. Mao, and W. Zhang, "An upper bound on transmission capacity of wireless CSMA networks," in Proceedings of the 2012 IEEE Global Communications Conference, GLOBECOM 2012, pp. 458-463, December 2012.

[29] H. Elsawy and E. Hossain, "A modified hard core point process for analysis of random CSMA wireless networks in general fading environments," IEEE Transactions on Communications, vol. 61, no. 4, pp. 1520-1534, 2013.

[30] G. Alfano, M. Garetto, and E. Leonardi, "New directions into the stochastic geometry analysis of dense CSMA networks," IEEE Transactions on Mobile Computing, vol. 13, no. 2, pp. 324-326, 2014.

[31] M. Nakagami, "The m-distribution, a general formula of intensity of rapid fading," in Statistical Methods in Radio Wave Propagation: Proceedings of a Symposium, pp. 3-36, 1958.

[32] I. Sen and D. W. Matolak, "Vehicle-vehicle channel models for the 5-GHz band," IEEE Transactions on Intelligent Transportation Systems, vol. 9, no. 2, pp. 235-245, 2008.

[33] "Cisco Aironet 1040 Series Wireless Access Point," https://www .cisco.com/c/en/us/products/wireless/aironet-1040-series/index .html.

[34] G. Pei and T. R. Henderson, "Validation of ns-3 802.11b PHY model," 2009, https://www.nsnam.org/ pei/80211b.pdf. 
[35] G. Pei and T. R. Henderson, "Validation of OFDM error rate model in ns-3," 2010, https://www.nsnam.org/ pei/80211ofdm .pdf.

[36] Y. Wang, "Simulation codes for this paper," 2016, https://github .com/marcherwang/wcmc_sim_codes. 


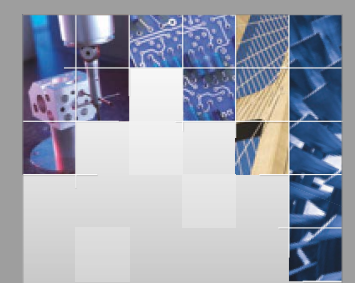

\section{Enfincering}
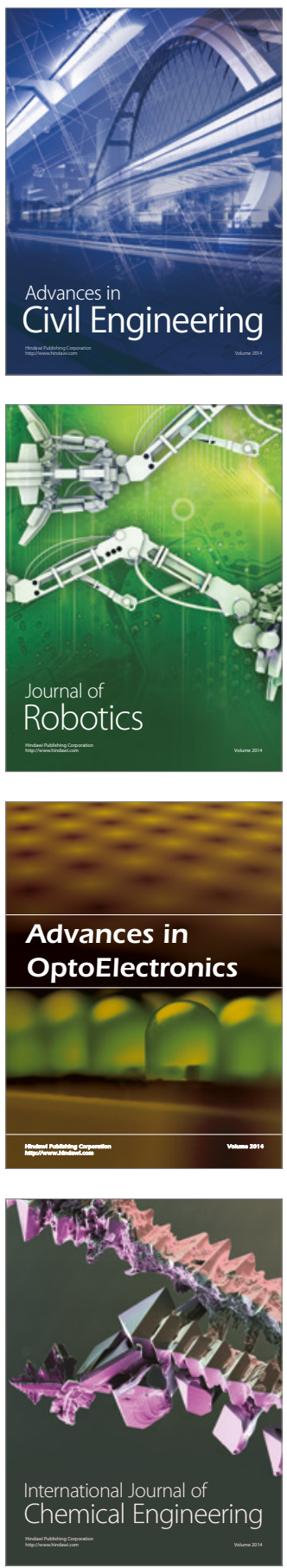

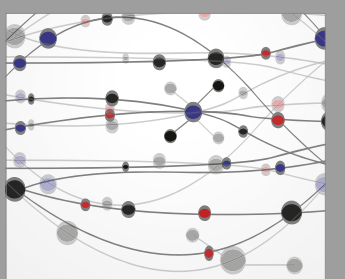

The Scientific World Journal

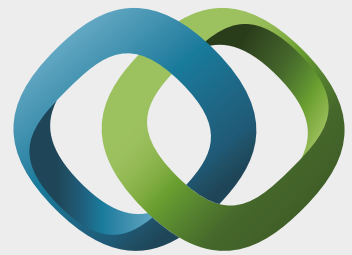

\section{Hindawi}

Submit your manuscripts at

https://www.hindawi.com
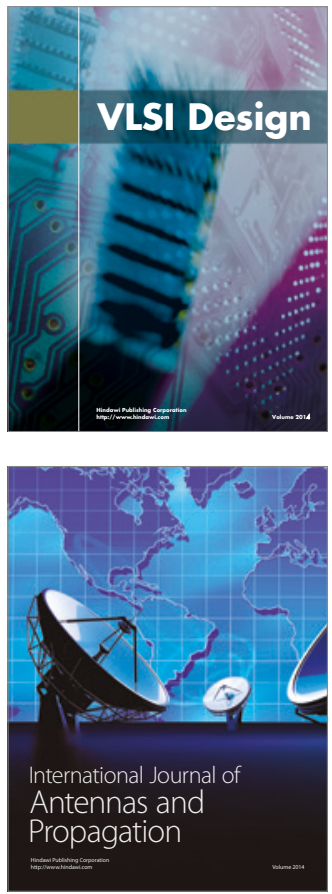

\section{Rotating}

Machinery
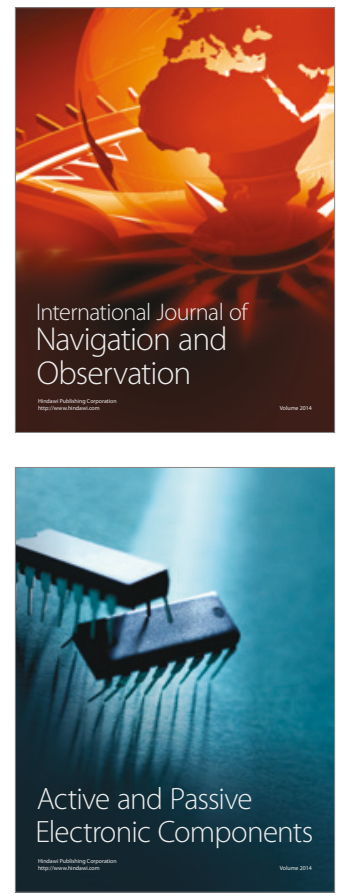
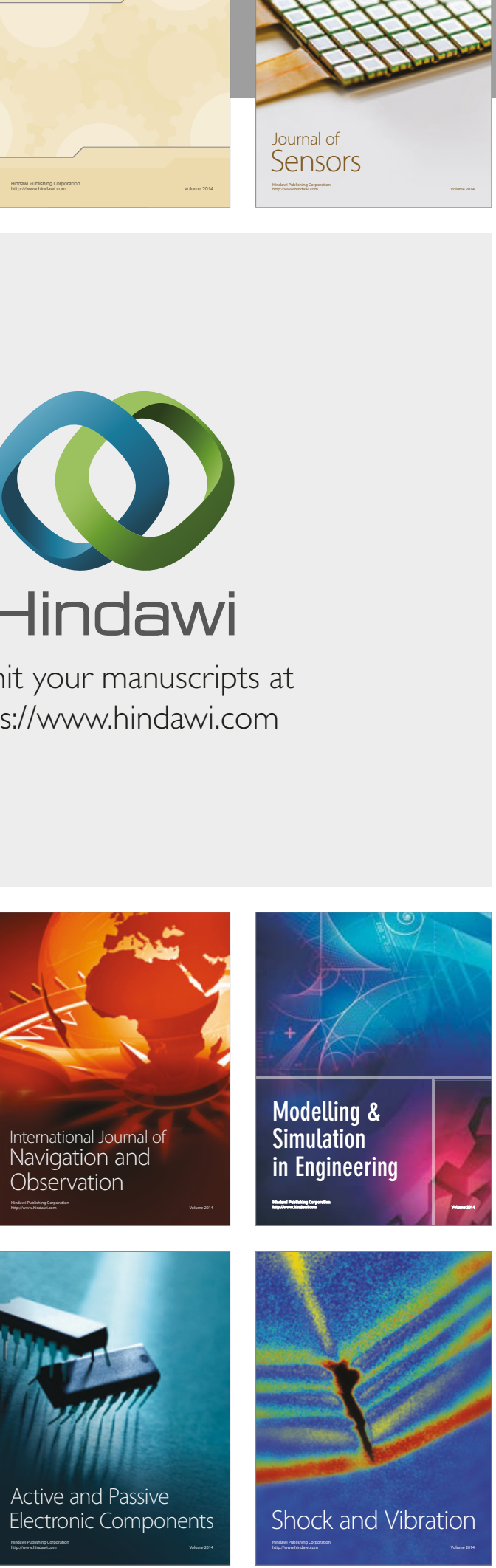
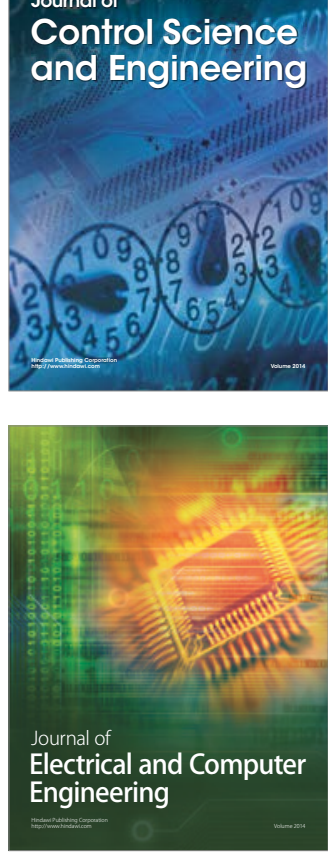

Distributed

Journal of

Control Science

and Engineering
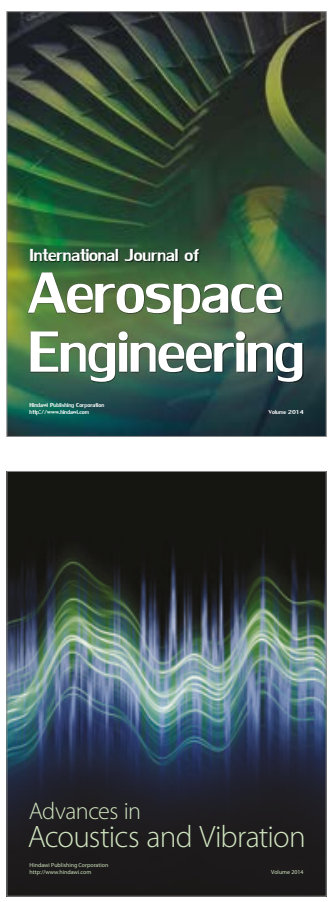

Sensor Networks 